\title{
Modelling and forecasting the demand for Thai tourism
}

\author{
H AIYAN S ONG, S TEPHEN F. W ITT AND G ANG LI \\ School of Management, University of Surrey, Guildford GU2 7XH, UK. \\ Tel: +441483 686353.Fax: +44 1483 686301.E-mail: h.song@surrey.ac.uk.
}

This study examines the demand for Thai tourism by seven major origin countries - Australia, Japan, Korea, Singapore, Malaysia, the UK and the USA. The general-to-specific modelling approach is followed in the construction, estimation, testing and selection of the tourism demand models. The empirical results show that habit persistence is the most important factor that influences the demand for Thai tourism by residents from all origin countries. The income, own price, cross price and trade volume variables are also found to be significant in the demand models, but the explanatory power of these variables, judged by the number of times they appear in the models, varies from origin to origin. The Asian financial crisis that occurred in late 1997 and early 1998 also appears to have had a significant impact on tourist arrivals from Singapore, Malaysia, Korea and the UK, but the magnitude and direction of influence are not the same for all models. The models that performed relatively well for each of the origin countries, according to both economic and statistical criteria, are selected to generate ex ante forecasts for the period up to 2010. The results suggest that Korea, Malaysia and Japan are expected to be the largest tourism generating countries by the end of the forecasting period, while the growth rate of tourist arrivals from Korea to Thailand is likely to be the highest among the seven origin countries.

Keywords: tourism demand; econometric model; forecasting; Thailand

Thailand was one of the first Asian countries to develop international tourism strategically as an industry, and has become the third largest tourist-receiving country within the Asia-Pacific region. Because of its vast number of tourist attractions, culture and strategic position, Thailand has been an important tourist destination for more than 150 years ( $\mathrm{Li}$ and Zhang, 1997). Tourism had developed without much intervention from the Thai government until the end of the 1970s, when the government realized the great contribution of tourism growth to the national economy, and began to incorporate its development into the national plan. In 1982 the tourism industry became the largest source of foreign exchange earnings for the first time. Since the 1980s the tourism industry has experienced rapid growth, and tourist arrivals have increased from 
1.86 million in 1980 to 9.51 million in $2000,{ }^{1}$ giving an $8.5 \%$ annual increase over this period. Meanwhile, tourism receipts have risen from 17.8 billion baht (US\$868 million) in 1980 to 285 billion baht (US\$7,119) in $2000 .{ }^{2}$ During the 1980s promotional campaigns were launched by the Thai government, and these included the 'Visit Thailand Year' campaign together with the Celebration of His Majesty the King's 60th Birthday Anniversary in 1987.

In the late 1990s, the Asian financial crisis had a significant impact on the national economy as well as on the Thai tourism industry. The impact on the tourism industry was double-sided. On the one hand, it resulted in the collapse of travel agents and tour operators in Japan and Korea; steep increases in exit taxes in Indonesia; fare wars throughout the region; airline lay-offs in Hong Kong; hotel redundancies; and a proposed (but later shelved) increase in petrol prices in Thailand. Such impacts were immediately felt in late 1997, when the crisis started. On the other hand, the currency devaluation resulted in a cost advantage for Thai tourism over alternative destinations. A surge of tourist arrivals from the European and North America markets occurred in early 1998. To some extent, this offset the loss incurred due to the drop in tourist arrivals from the Asian markets. As Higham (2000, p 101) states, 'tourism is widely recognized as the sector most likely to lead a recovery'.

Considering the essential role that the Thai tourism industry plays in its national economy, accurate forecasts of international tourism demand can provide vital information for both policy determination by the government (such as taxes and subsidies) and strategic planning by the private sector. In terms of analysing and forecasting tourism demand, Thailand has attracted very little attention. The study by Vogt and Wittayakorn (1998) is the only econometric analysis of the demand for Thai tourism. However, the modelling strategy and forecasting performance of the demand models developed in their study were not fully and systematically explored. The empirical results generated from their study are therefore subject to much criticism for the lack of theoretical foundation. This paper aims to apply a more rigorous modelling strategy known as the 'generalto-specific' approach developed by economists in the early 1990s (see Hendry, 1995) to investigate systematically the determinants of the demand for Thai tourism. The models estimated using this methodology are also evaluated by examining their forecasting performance. Although Song and Witt $(2000 ; 2003)$ have recently introduced this approach to tourism demand analysis, their studies focus mainly on the theoretical aspects of the methodology, and the appropriateness of this methodology was tested using data relating only to a small number of origin-destination pairs. The current research, however, extends Song and Witt's work by including more origin-destination pairs in the empirical study. The contribution of this paper is twofold. First, it adds additional evidence to the literature in supporting the use of the general-to-specific approach to model and forecast tourism demand. Second, the empirical findings should provide useful information for decision makers and planners in Thailand.

The remainder of the paper is organized as follows. The next section summarizes the general-to-specific modelling methodology. The third section discusses the definitions of the data and shows the preliminary analysis of the variables used. Empirical modelling results and ex ante forecasts for the period up to 2010 are given in the fourth section. The last section summarizes and draws conclusions. 


\section{Methodology}

Most tourism demand modelling and forecasting studies published before the 1990s are classical regression analyses, with ordinary least squares (OLS) as the main estimation procedure. The demand models are normally specified as loglinear single-equation models. Economic theory is used to recommend what variables should be included in the demand models, while simple hypothesis testing statistics, such as the $t$-statistic and $F$-statistic based on the OLS estimates, are used to determine whether or not an individual explanatory variable or all explanatory variables is/are significant as determinants of tourism demand. However, many of these studies do not pay much attention to providing a sound and consistent theoretical framework for tourism demand modelling. If the initial model suffers from autocorrelation or heteroscedasticity, which is an indication of model mis-specification, the modeller would 'add or subtract variables, change the definition of variables and so forth' on an ad hoc basis in order to solve the mis-specification problem (Gilbert, 1986, p 284). Such a methodology is called the 'simple-to-general' modelling approach. This approach does not have a clear modelling strategy and is often criticized for its copious data mining.

In contrast to this traditional approach a modern econometric methodology, the 'general-to-specific' approach, is attracting more and more attention. Within the general-to-specific framework, the specification starts from a general autoregressive distributed lag model (ADLM), which incorporates as many variables as possible supported by appropriate economic theory, and takes the form:

$$
y_{t}=\alpha_{0}+\sum_{j=1}^{k} \sum_{i=0}^{l} \alpha_{j i} x_{j t-i}+\sum_{i=1}^{l} \beta_{i} y_{t-i}+\text { dummies }+\varepsilon_{t}
$$

where $y$ and $x$ s are dependent (tourism demand in our case) and explanatory variables, respectively; $l$ is the lag length, $k$ is the number of explanatory variables, and $\alpha$ s and $\beta$ s are parameters that need to be estimated. By imposing different restrictions on the parameters, various specific models may be obtained from the general ADLM. These comprise the autoregressive, static, growth rate, partial adjustment, dead start, leading indicator, common factor, finite distributed lag and error correction models. Diagnostic tests are carried out to select the best performing model. The application of the general-to-specific modelling approach to tourism forecasting is described in detail in Song and Witt (2003), and is summarized here.

The general ADLM is first estimated and the sum of squared residuals of the general model is calculated. Then the restricted (specific) model is estimated and the sum of the squared residuals of this model is calculated. The third step is to test the restrictions imposed by comparing the sums of squared residuals of the ADLM and the restricted model using the $F$-statistic. Since the restricted model is simple in structure and has more degrees of freedom than the general ADLM when it is estimated, the specific model is preferred to the complicated ADLM if the restrictions are accepted.

One of the advantages of the general ADLM is that a modern econometric technique, known as error correction, can be readily incorporated into the modelling process. The error correction model in the tourism context is based 
on the assumption that tourists make rational decisions on the demand for tourism at time $t$ using all the information available (income, price, substitute prices, and so on) in the long run, but make occasional decision errors in purchasing tourism products in the short run due to information asymmetry. As a result of the decision errors made by tourists the demand for tourism in the short run deviates from its long-run equilibrium path (or steady state). ${ }^{3}$ This deviation is not sustainable over time because tourists, as rational agents, learn from their mistakes and remove their decision errors in order to achieve the long-run equilibrium demand. Therefore, the demand for tourism as a dynamic process is self-correcting. Engle and Granger (1987) termed this self-correction process an 'error correction mechanism', and demonstrated that the process may be modelled using the error correction model (ECM).

The ECM may be estimated in different ways: the most popular of these are the Wickens-Breusch approach (WB) (Wickens and Breusch, 1988) and the Johansen maximum likelihood method (JML) (Johansen, 1988). The WickensBreusch approach is particularly appropriate for small samples, but assumes that there is only one cointegration (CI) relationship among all the variables in the demand equation, and, consequently, only one error correction model may be formulated. (When multiple CI relationships exist, they are 'averaged' into a single vector.) The Johansen approach allows for multiple CI relationships to be identified. Subsequently, multiple ECMs are obtained and the number of ECMs equals the number of CI relationships.

To evaluate the performance of each specific model and select the appropriate ones for forecasting, various diagnostic tests need to be carried out. These tests, as used by applied econometricians, include the Breusch (1978) and Godfrey (1978) Lagrange Multiplier chi-square test for serial correlation, the JarqueBera (1980) chi-square test for non-normality, the Ramsey (1969) RESET test for mis-specification, the White (1980) chi-square test for heteroscedasticity and the Chow (1960) test for predictive failure. In addition, the ex post forecast accuracy should also be checked, given sufficient observations.

To forecast tourism demand using multivariate regression models, various independent variables need to be forecast first. Following Song and Witt (2003), this paper uses the Holt-Winter two-parameter exponential smoothing technique to forecast the influencing factors involved, and single-parameter method for error correction terms in the WB and JML ECMs.

In assessing the forecasting performance of the selected econometric models, ARIMA models are employed as benchmarks. ${ }^{4}$

\section{Data description}

The empirical study in this paper is based on inbound tourism demand to Thailand from seven major international markets - Australia, Japan, Korea, Malaysia, Singapore, the UK and the USA. ${ }^{5}$ Total tourist arrivals from these seven countries accounted for more than $50 \%$ of total international tourist arrivals in $2000 .^{6}$

The dependent variable, tourism demand, is measured by tourist arrivals in year $t,{ }^{7}$ denoted as $T O U_{t}$. Independent variables include income $\left(G D P_{t}\right)$, relative tourism price $\left(R R C P_{t}\right)$, substitute tourism price $\left(R S U B_{t}\right)$, trade volume $\left(T R A_{t}\right)$ 
and some dummy variables. $G D P_{t}$ is measured by GDP in 1995 prices; $R R C P_{t}$ is measured by the CPI $(1995=100)$ in Thailand relative to that in origin country $j$, adjusted by the relevant exchange rate; $R S U B_{t}$ is measured by a weighted average price index of alternative destinations relative to the tourism price in the origin country. ${ }^{8}$ Singapore, Indonesia and the Philippines are chosen as alternative destinations for Malaysia; Malaysia, Indonesia and the Philippines for Singapore; Singapore, Malaysia, the Philippines and Indonesia for the other origins. TRA $A_{t}$ is measured by the sum of import and export volumes between the origin country and Thailand, adjusted by import and export price indices (or unit values, 1995=100) respectively, to generate the constant price form. All of these data have been transformed into logarithms, denoted as the letter $L$ in front of the variable names.

Concerning the effects of one-off events on the demand for Thai tourism, dummy variables are included in the original models. DUM74 and DUM79 are specified to capture the effects of the two oil crises (DUM74=1 in 1974 and 0 otherwise; DUM79=1 in 1979 and 0 otherwise). DUM87 reflects the influence of the 'Visit Thailand Year' campaign in 1987 (DUM87=1 in 1987 and 1988 and 0 otherwise). Given that the effect may be different between years, two dummies are specified to capture the influence of the Asian financial crisis which arose in the middle of 1997, and continued into 1998. DUM97 (= 1 in 1997 and 0 otherwise) detects the influence in 1997, and DUM98 (=1 in 1998 and 0 otherwise) in the following year. The Seoul Olympics in 1988 and the student demonstrations in 1980 are expected to have reduced Korea's outbound tourism to Thailand during the years concerned. Therefore, DUM88 (=1 in 1988 and 0 otherwise) and DUM80 (=1 in 1980 and 0 otherwise) are considered in the Korean models to examine the effects of the two events, respectively. ${ }^{9}$

The sample of data covers 1963-2000 for Australia and the UK, and 19682000 for the other countries. Tourist arrivals before 1978 are collected from the Annual Reports of the Tourism Authority of Thailand (TAT), and the series after 1978 are from the Tourism Statistical Yearbook, published by the World Tourism Organization (WTO). Data on GDP, exchange rates, CPI, import and export price indices (or unit values) are obtained from International Financial Statistics Yearbook published by the International Monetary Fund (IMF). The figures on imports and exports are obtained from Direction of Trade Statistics Yearbook published by the IMF.

\section{Empirical results}

\section{Estimates of the general models}

Tourism demand from each origin is modelled with the following ADLM based on the whole sample in order to examine the impacts of the financial crisis in 1997 and 1998:

$$
\begin{gathered}
\operatorname{LTOU}_{t}=\alpha_{0}+\alpha_{1} \text { LTOU }_{t-1}+\beta_{0} L G P_{t}+\beta_{1} L G D P_{t-1}+\gamma_{0} L R R C P_{t}+\gamma_{1} L_{R R C P_{t-1}} \\
+\theta_{0} \operatorname{LRSUB}_{t}+\theta_{1} L R S U B_{t-1}+\varphi_{0} L T R A_{t}+\varphi_{1} L T R A_{t-1}+\text { dummies }+\varepsilon_{\mathrm{t}} \\
(\mathrm{j}=1,2, \ldots, 7)
\end{gathered}
$$




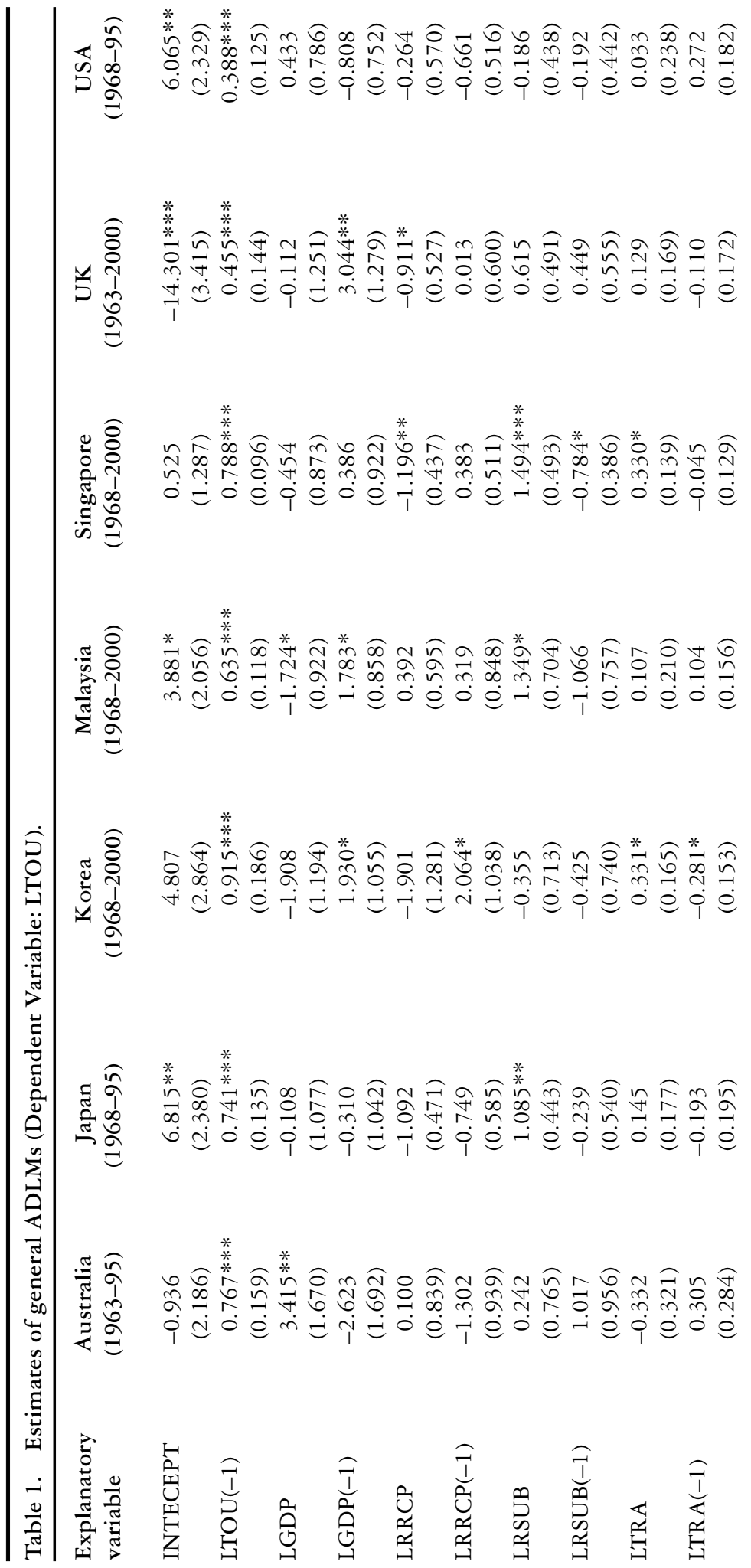




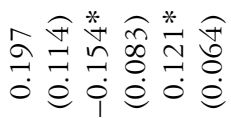

$\begin{array}{lll}\hat{0} & \hat{0} & n \\ \vdots & 0 & 0 \\ 0 & 0\end{array}$

๙

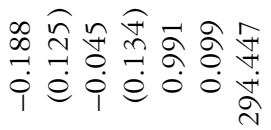

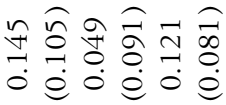

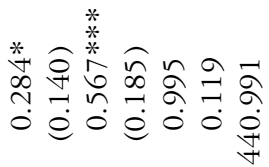

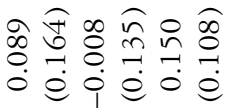

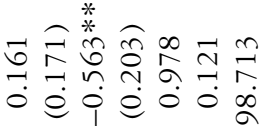

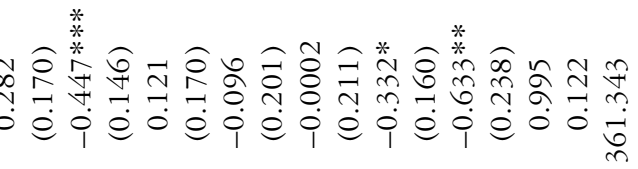

|

i $\dot{0} \dot{0} \dot{0} \dot{0} \dot{0}$

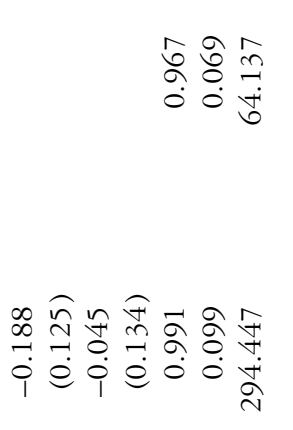

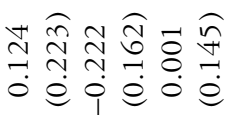

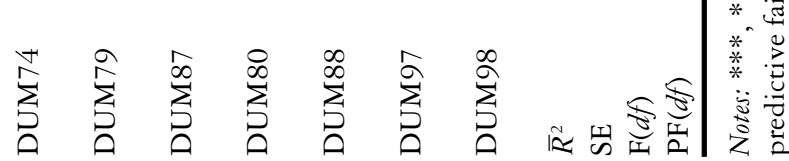


Table 2. Restriction test results (F-test).

\begin{tabular}{|c|c|c|c|c|c|c|c|}
\hline Specific model & Australia & Japan & Korea & Malaysia & Singapore & UK & USA \\
\hline Static & 7.457 & 14.065 & 7.451 & 10.600 & 29.173 & 7.339 & 2.983 \\
\hline Autoregressive & $1.781 * *$ & 4.671 & 2.751 & 2.909 & 30.66 & 3.166 & 4.866 \\
\hline Growth rate & $1.652 * *$ & 6.264 & $1.019 * *$ & 4.480 & 5.140 & 4.910 & 7.122 \\
\hline $\begin{array}{l}\text { Leading } \\
\text { indicator }\end{array}$ & 5.208 & 6.746 & 8.437 & 10.815 & 21.735 & 3.759 & 3.119 \\
\hline $\begin{array}{l}\text { Partial } \\
\text { adjustment }\end{array}$ & $1.233 * *$ & 4.246 & $1.639 * *$ & $2.673 * *$ & $1.608 * *$ & $2.445 * *$ & $1.409 * *$ \\
\hline $\begin{array}{l}\text { Finite } \\
\text { distributed lag }\end{array}$ & 23.226 & 30.282 & 24.262 & 28.866 & 68.010 & 9.932 & 9.622 \\
\hline Dead start & $1.285 * *$ & $1.595 * *$ & 3.441 & $2.909 * *$ & 3.539 & $0.998 * *$ & $0.474 * *$ \\
\hline
\end{tabular}

Note: $* *$ indicates that the specific model is accepted at the $5 \%$ level.

In the cases of Australia, Japan and the USA, where no influence of the Asian financial crisis was detected, the general models are re-estimated using data up to 1995 , leaving the remaining data for the ex post forecasting evaluation using the Chow (1960) predictive failure test. This test is not applied to the other four countries, as demand was clearly affected by the financial crisis and this would invalidate the test automatically. The estimation results are shown in Table 1.

The lagged dependent variable seems to be the most important determinant of the demand for Thai tourism, and is significant in all models at the $1 \%$ significance level. It indicates that the demand for Thai tourism features a stable behaviour pattern, or 'habit persistence', and that the 'word of mouth' effect plays an important role in the determination of tourists' choice of Thailand as a tourist destination. Furthermore, the presence of the lagged dependent variable in the partial adjustment model (shown in Tables $3,5,6,7,8$ and 9) is also justified on the grounds of supply constraints (Witt, 1980). Income and own price (either lagged or current variables) are the second most vital determinants, both being significant in four out of seven cases (at least at the $10 \%$ level). The trade volume and cross price variables are also significant in some cases. The signs of the coefficients for the cross price variable show that the chosen alternative destinations appear to be substitutes for Thailand in the Australia, Japan, Singapore, Malaysia and UK models, and complements in the Korea and USA models. This suggests that tourists from Korea and the USA prefer to visit Thailand along with other neighbouring countries during the same trip, while tourists from the other origins are more likely to choose between Thailand and the alternative destinations.

With regard to the dummy variables, the significance varies from one model to another. The Asian financial crisis seems to have had the most widespread impacts on the demand for Thai tourism among the origin countries, as the financial crisis dummies are found to be significant in four out of seven origin countries (including Malaysia, where the effect is not significant in the general model but is significant in most of the specific models). 


\section{Restriction tests}

Table 2 presents the results for the restriction $F$ tests. The partial adjustment model, as a result of imposing the restriction $\beta_{1}=\gamma_{1}=\theta_{1}=\varphi_{1}=0$ on the ADLM (Equation 2) seems to be the most prevailing functional form in this study (accepted by all of the origins except Japan), followed by the dead start model $\left(\beta_{0}=\gamma_{1}=\theta_{0}=\varphi_{0}=0\right.$ in the ADLM), growth rate model $\left(\alpha_{1}=1, \beta_{0}=-\beta_{1}\right.$,

Table 3. Estimates of Australia models (1963-95).

\begin{tabular}{|c|c|c|c|c|c|c|}
\hline & \multicolumn{3}{|c|}{ Dependent variable: LTOU } & \multicolumn{3}{|c|}{ Dependent variable: DLTOU } \\
\hline & $\begin{array}{c}\text { Dead } \\
\text { start }\end{array}$ & $\begin{array}{c}\text { Partial } \\
\text { adjustment }\end{array}$ & $\begin{array}{c}\text { Reduced } \\
\text { ADLM }\end{array}$ & & WB-ECM & JML-ECM \\
\hline INTECEPT & $\begin{array}{c}0.224 \\
(1.982)\end{array}$ & $\begin{array}{c}0.790 \\
(1.960)\end{array}$ & $\begin{array}{c}-0.194 \\
(1.563)\end{array}$ & INTECEPT & $\begin{array}{c}-1.028 \\
(1.660)\end{array}$ & $\begin{array}{c}-0.165 \\
(0.112)\end{array}$ \\
\hline LTOU $(-1)$ & $\begin{array}{l}0.630^{* * *} \\
(0.141)\end{array}$ & $\begin{array}{l}0.674 * * * \\
(0.121)\end{array}$ & $\begin{array}{l}0.589 * * * \\
(0.120)\end{array}$ & DLGDP & $\begin{array}{l}3.205 * * \\
(1.400)\end{array}$ & $\begin{array}{c}2.693 \\
(1.677)\end{array}$ \\
\hline LGDP & & $\begin{array}{c}1.019 * \\
(0.563)\end{array}$ & $\begin{array}{l}1.446 * * * \\
(0.502)\end{array}$ & DLRRCP & & $\begin{array}{c}0.222 \\
(0.726)\end{array}$ \\
\hline $\operatorname{LGDP}(-1)$ & $\begin{array}{c}0.802 \\
(0.594)\end{array}$ & & & DLRSUB & & $\begin{array}{c}-0.025 \\
(0.717)\end{array}$ \\
\hline LRRCP & & $\begin{array}{c}-0.810 \\
(0.634)\end{array}$ & & LTOU $(-1)$ & $\begin{array}{c}-0.324 * * \\
(0.135)\end{array}$ & \\
\hline $\operatorname{LRRCP}(-1)$ & $\begin{array}{r}-1.274^{*} \\
(0.734)\end{array}$ & & $\begin{array}{c}-1.472 * * \\
(0.543)\end{array}$ & LGDP(-1) & $\begin{array}{l}1.208 * * \\
(0.525)\end{array}$ & \\
\hline LRSUB & & $\begin{array}{c}0.950 \\
(0.626)\end{array}$ & & LRRCP(-1) & $\begin{array}{c}-1.494 * * * \\
(0.535)\end{array}$ & \\
\hline $\operatorname{LRSUB}(-1)$ & $\begin{array}{c}1.445 * \\
(0.772)\end{array}$ & & $\begin{array}{l}1.686 * * * \\
(0.553)\end{array}$ & LRSUB(-1) & $\begin{array}{l}1.696 * * * \\
(0.545)\end{array}$ & \\
\hline LTRA & & $\begin{array}{c}0.017 \\
(0.106)\end{array}$ & & $\operatorname{ECM}(-1)$ & & $\begin{array}{c}-0.052 \\
(0.036)\end{array}$ \\
\hline $\operatorname{LTRA}(-1)$ & $\begin{array}{c}0.189 \\
(0.118)\end{array}$ & & & & & \\
\hline DUM 74 & $\begin{array}{c}0.077 \\
(0.218)\end{array}$ & $\begin{array}{c}0.283 \\
(0.176)\end{array}$ & & & & \\
\hline DUM79 & $\begin{array}{c}-0.182 \\
(0.158)\end{array}$ & $\begin{array}{c}-0.189 \\
(0.159)\end{array}$ & & & & \\
\hline DUM87 & $\begin{array}{c}0.104 \\
(0.135)\end{array}$ & $\begin{array}{c}0.052 \\
(0.123)\end{array}$ & & & & \\
\hline $\bar{R}^{2}$ & 0.977 & 0.978 & 0.979 & $\bar{R}^{2}$ & 0.437 & 0.208 \\
\hline SE & 0.152 & 0.152 & 0.147 & SE & 0.145 & 0.172 \\
\hline $\mathrm{SC}(2)$ & 2.043 & 0.752 & 0.019 & $\mathrm{SC}(2)$ & 0.027 & 0.149 \\
\hline $\mathrm{FF}(1)$ & 0.746 & 0.0001 & 0.005 & $\mathrm{FF}(1)$ & 3.542 & 0.001 \\
\hline $\mathrm{NO}(2)$ & 1.581 & 2.922 & 0.419 & $\mathrm{NO}(2)$ & 0.531 & 0.866 \\
\hline $\mathrm{HE}(1)$ & 10.638 & 13.242 & 8.802 & $\operatorname{HE}(1)$ & 10.241 & 9.969 \\
\hline $\operatorname{PF}(d f)$ & 0.154 & 0.293 & 0.220 & $\mathrm{PF}(d f)$ & 0.222 & 0.378 \\
\hline MAPE & 0.473 & 0.611 & 1.073 & MAPE & 1.016 & 1.484 \\
\hline RMSE & 0.078 & 0.091 & 0.145 & RMSE & 0.142 & 0.220 \\
\hline
\end{tabular}

Notes: see Table 1. SC(2) is the Lagrange multiplier test for serial correlation; $\mathrm{NO}(2)$ is the Jarque-Bera normality test; $\mathrm{FF}(1)$ is Ramsey's mis-specification test; $\mathrm{HE}(1)$ is a heteroscedasticity test; ARCH is the autoregressive conditional heteroscedasticity test. All statistics are Chi-square statistics. 
$\gamma_{0}=-\gamma_{1}, \theta_{0}=-\theta_{1}$ and $\varphi_{0}=-\varphi_{1}$ in the ADLM) and the autoregressive model $\left(\beta_{0}=\beta_{1}=\gamma_{0}=\gamma_{1}=\theta_{0}=\theta_{1}=\varphi_{0}=\varphi_{1}=0\right.$ in the ADLM $)$. The static $\left(\alpha_{1}\right.$ $=\beta_{1}=\gamma_{1}=\theta_{1}=\varphi_{1}=0$ in the ADLM $)$, leading indicator $\left(\beta_{0}=\gamma_{0}=\theta_{0}=\right.$ $\varphi_{0}=0$ in the ADLM) and finite distributed lag $\left(\alpha_{1}=0\right.$ in the ADLM) models do not seem to be valid for any of the origin countries.

\section{Estimates of the restricted models and forecasts}

All of the accepted specific models are estimated, along with another specific model that is achieved based on a modelling process known as the variable reduction process. This reduction process filters out insignificant variables from

Table 4. Estimates of Japan models (1969-95).

\begin{tabular}{|c|c|c|c|c|c|}
\hline & \multicolumn{2}{|c|}{ Dependent variable: LTOU } & & \multicolumn{2}{|c|}{ Dependent variable: DLTOU } \\
\hline & Dead start & Reduced ADLM & & WB-ECM & JML-ECM \\
\hline INTECEPT & $\begin{array}{l}5.790 * * * \\
(1.615)\end{array}$ & $\begin{array}{l}4.993 * * * \\
(1.352)\end{array}$ & INTECEPT & $\begin{array}{l}5.203 * * * \\
(1.735)\end{array}$ & $\begin{array}{c}-0.005 \\
(0.023)\end{array}$ \\
\hline $\operatorname{LTOU}(-1)$ & $\begin{array}{l}0.588 * * * \\
(0.069)\end{array}$ & & DLRRCP & & $\begin{array}{c}-0.725 * \\
(0.371)\end{array}$ \\
\hline LGD & & & DLRSUB & $\begin{array}{c}0.167 \\
(0.178)\end{array}$ & $\begin{array}{l}0.821 * * \\
(0.342)\end{array}$ \\
\hline $\operatorname{LGDP}(-1)$ & & & LTOU $(-1)$ & $\begin{array}{c}-0.403 * * * \\
(0.070)\end{array}$ & \\
\hline LRRCP & & $\begin{array}{c}-0.709 * * \\
(0.314)\end{array}$ & $\operatorname{LGDP}(-1)$ & & \\
\hline $\operatorname{LRRCP}(-1)$ & $\begin{array}{c}-1.295 * * * \\
(0.380)\end{array}$ & & $\operatorname{LRRCP}(-1)$ & $\begin{array}{c}-1.475 * * * \\
(0.426)\end{array}$ & \\
\hline LRSUB & & $\begin{array}{l}0.772 * * \\
(0.320)\end{array}$ & LRSUB(-1) & $\begin{array}{c}0.642 \\
(0.438)\end{array}$ & \\
\hline $\operatorname{LRSUB}(-1)$ & $\begin{array}{c}0.444 \\
(0.383)\end{array}$ & & DUM74 & $\begin{array}{c}-0.312 * * \\
(0.113)\end{array}$ & $\begin{array}{c}-0.286 * * \\
(0.087)\end{array}$ \\
\hline LTRA & & & DUM79 & $\begin{array}{c}-0.232 * * \\
(0.091)\end{array}$ & $\begin{array}{c}-0.198 * * \\
(0.084)\end{array}$ \\
\hline $\operatorname{LTRA}(-1)$ & & & $\operatorname{ECM}(-1)$ & & $\begin{array}{c}-0.389 * * * \\
(0.051)\end{array}$ \\
\hline DUM74 & $\begin{array}{c}-0.271 * * \\
(0.104)\end{array}$ & $\begin{array}{c}-0.258 * * * \\
(0.088)\end{array}$ & & & \\
\hline DUM79 & $\begin{array}{c}-0.215 * * \\
(0.089)\end{array}$ & $\begin{array}{c}-0.202 * * \\
(0.086)\end{array}$ & & & \\
\hline DUM87 & & & & & \\
\hline $\bar{R}^{2}$ & 0.987 & 0.989 & $\bar{R}^{2}$ & 0.650 & 0.711 \\
\hline SE & 0.083 & 0.077 & SE & 0.083 & 0.076 \\
\hline $\mathrm{SC}(2)$ & 0.908 & 0.174 & $\mathrm{SC}(2)$ & 0.225 & 0.164 \\
\hline $\mathrm{FF}(1)$ & 1.943 & 1.338 & $\mathrm{FF}(1)$ & 1.065 & 0.002 \\
\hline $\mathrm{NO}(2)$ & 2.772 & 0.798 & $\mathrm{NO}(2)$ & 2.188 & 0.775 \\
\hline $\mathrm{HE}(1)$ & 10.872 & 1.322 & $\mathrm{HE}(1)$ & 13.335 & $15.586 * *$ \\
\hline $\operatorname{PF}(d f)$ & 1.378 & 1.322 & $\mathrm{PF}(d f)$ & 1.431 & 1.700 \\
\hline MAPE & 1.660 & 1.634 & MAPE & 1.673 & 2.828 \\
\hline RMSE & 0.242 & 0.233 & RMSE & 0.247 & 0.429 \\
\hline
\end{tabular}

Note: see Tables 1 and 3. 
Table 5 . Estimates of Korea models (1968-2000).

\begin{tabular}{|c|c|c|c|c|c|c|}
\hline & \multicolumn{2}{|c|}{ Dependent variable: LTOU } & & \multicolumn{3}{|c|}{ Dependent variable: DLTOU } \\
\hline & $\begin{array}{c}\text { Partial } \\
\text { adjustment }\end{array}$ & $\begin{array}{l}\text { Reduced } \\
\text { ADLM }\end{array}$ & & $\begin{array}{l}\text { Growth } \\
\text { rate }\end{array}$ & WB-ECM & JML-ECM \\
\hline INTECEPT & $\begin{array}{c}2.977 \\
(2.631)\end{array}$ & $\begin{array}{c}1.173 \\
(1.770)\end{array}$ & INTECEPT & $\begin{array}{l}0.298 * * * \\
(0.078)\end{array}$ & $\begin{array}{c}0.839 \\
(1.779)\end{array}$ & $\begin{array}{c}-0.306 \\
(0.311)\end{array}$ \\
\hline LTOU(-1) & $\begin{array}{l}0.724 * * * \\
(0.112)\end{array}$ & $\begin{array}{l}0.672 * * * \\
(0.083)\end{array}$ & DLGDP & $\begin{array}{l}-2.012 * * \\
(0.913)\end{array}$ & & $\begin{array}{c}-0.881 \\
(0.813)\end{array}$ \\
\hline LGDP & $\begin{array}{l}0.455 * * \\
(0.215)\end{array}$ & & DLRRCP & $\begin{array}{c}-2.185 * * \\
(0.817)\end{array}$ & & \\
\hline $\operatorname{LGDP}(-1)$ & & $\begin{array}{l}0.671 * * * \\
(0.181)\end{array}$ & DLRSUB & $\begin{array}{c}0.041 \\
(0.533)\end{array}$ & $\begin{array}{c}-1.161 * * * \\
(0.290)\end{array}$ & $\begin{array}{c}-1.330 * * * \\
(0.331)\end{array}$ \\
\hline LRRCP & & & DLTRA & $\begin{array}{c}0.290 * * \\
(0.121)\end{array}$ & & \\
\hline $\operatorname{LRRCP}(-1)$ & & & $\operatorname{LTOU}(-1)$ & & $\begin{array}{c}-0.274 * * * \\
(0.094)\end{array}$ & \\
\hline LRSUB & $\begin{array}{c}-0.094 * * * \\
(0.266)\end{array}$ & $\begin{array}{c}-0.952 * * * \\
(0.233)\end{array}$ & $\operatorname{LGDP}(-1)$ & & $\begin{array}{l}0.569 * * * \\
(0.199)\end{array}$ & \\
\hline $\operatorname{LRSUB}(-1)$ & & & $\operatorname{LRRCP}(-1)$ & & & \\
\hline LTRA & $\begin{array}{c}0.055 \\
(0.102)\end{array}$ & & LRSUB(-1) & & $\begin{array}{c}-0.781 * * * \\
(0.272)\end{array}$ & \\
\hline $\operatorname{LTRA}(-1)$ & & & DUM74 & $\begin{array}{c}0.162 \\
(0.147)\end{array}$ & & \\
\hline DUM74 & $\begin{array}{c}0.144 \\
(0.134)\end{array}$ & & DUM79 & $\begin{array}{l}-0.414 * * * \\
(0.132)\end{array}$ & $\begin{array}{c}-0.332 * * \\
(0.129)\end{array}$ & $\begin{array}{c}-0.389^{* *} \\
(0.145)\end{array}$ \\
\hline DUM79 & $\begin{array}{c}-0.310 * * \\
(0.132)\end{array}$ & $\begin{array}{c}-0.312 * * \\
(0.130)\end{array}$ & DUM87 & $\begin{array}{c}0.066 \\
(0.128)\end{array}$ & & \\
\hline DUM87 & & & DUM80 & $\begin{array}{c}-0.009 \\
(0.168)\end{array}$ & & \\
\hline DUM80 & $\begin{array}{c}-0.119 \\
(0.133)\end{array}$ & & DUM88 & $\begin{array}{c}0.056 \\
(0.196)\end{array}$ & & \\
\hline DUM88 & $\begin{array}{c}0.186 \\
(0.155)\end{array}$ & & DUM97 & $\begin{array}{c}-0.363 * * \\
(0.140)\end{array}$ & $\begin{array}{c}-0.280 * * \\
(0.135)\end{array}$ & $\begin{array}{c}-0.259 * \\
(0.148)\end{array}$ \\
\hline DUM97 & $\begin{array}{c}-0.267 * \\
(0.154)\end{array}$ & $\begin{array}{c}-0.284 * * \\
(0.136)\end{array}$ & DUM98 & $\begin{array}{c}-0.719 * * * \\
(0.201)\end{array}$ & $\begin{array}{c}-0.767 * * * \\
(0.138)\end{array}$ & $\begin{array}{c}-0.810 * * * \\
(0.185)\end{array}$ \\
\hline DUM98 & $\begin{array}{c}-0.671 * * * \\
(0.192)\end{array}$ & $\begin{array}{c}-0.792 * * * \\
(0.138)\end{array}$ & $\operatorname{ECM}(-1)$ & & & $\begin{array}{c}-0.132 * \\
(0.071)\end{array}$ \\
\hline $\bar{R}^{2}$ & 0.994 & 0.994 & $\bar{R}^{2}$ & 0.738 & 0.735 & 0.667 \\
\hline SE & 0.124 & 0.124 & SE & 0.122 & 0.123 & 0.137 \\
\hline $\mathrm{SC}(2)$ & 0.373 & 3.899 & $\mathrm{SC}(2)$ & 2.890 & 1.943 & 0.572 \\
\hline $\mathrm{FF}(1)$ & 1.188 & 1.919 & $\mathrm{FF}(1)$ & 0.655 & $6.544 * *$ & 1.887 \\
\hline $\mathrm{NO}(2)$ & 2.877 & 0.015 & $\mathrm{NO}(2)$ & 0.739 & 0.108 & 1.067 \\
\hline $\mathrm{HE}(1)$ & 7.798 & 8.441 & $\operatorname{HE}(1)$ & 11.118 & 10.422 & 14.964 \\
\hline
\end{tabular}

Note: see Tables 1 and 3.

the general model. The least significant variable is first deleted from the original ADLM, and the reduced model is then re-estimated. This process is repeated until all the remaining variables are significant according to the $t$ statistic, and have the correct signs. The specific model achieved using this process is termed a 'reduced ADLM'. The remaining variables in the reduced 
Table 6. Estimates of Malaysia models (1968-2000).

Dependent variable: LTOU

Partial Reduced ADLM adjustment
Dependent variable: DLTOU WB-ECM JML-ECM

\begin{tabular}{|c|c|c|c|c|c|}
\hline INTECEPT & $\begin{array}{c}1.745 \\
(1.806)\end{array}$ & $\begin{array}{l}2.561 * * * \\
(0.648)\end{array}$ & INTECEPT & $\begin{array}{l}2.375 * * * \\
(0.603)\end{array}$ & $\begin{array}{c}-0.192 * * \\
(0.087)\end{array}$ \\
\hline LTOU(-1) & $\begin{array}{l}0.630 * * * \\
(0.111)\end{array}$ & $\begin{array}{l}0.819 * * * \\
(0.046)\end{array}$ & DLGDP & & $\begin{array}{c}-1.609 * * \\
(0.691)\end{array}$ \\
\hline LGDP & $\begin{array}{c}0.127 \\
(0.272)\end{array}$ & & DLRRCP & & \\
\hline $\operatorname{LGDP}(-1)$ & & & DLRSUB & $\begin{array}{l}1.687 * * * \\
(0.555)\end{array}$ & $\begin{array}{l}1.572 * * * \\
(0.505)\end{array}$ \\
\hline LRRCP & $\begin{array}{c}-0.843 \\
(0.609)\end{array}$ & & LTOU(-1) & $\begin{array}{c}-0.297 * * * \\
(0.081)\end{array}$ & \\
\hline $\operatorname{LRRCP}(-1)$ & & & $\operatorname{LGDP}(-1)$ & $\begin{array}{c}0.238^{*} \\
(0.120)\end{array}$ & \\
\hline LRSUB & $\begin{array}{c}0.829 \\
(0.525)\end{array}$ & $\begin{array}{l}0.767 * * \\
(0.343)\end{array}$ & $\operatorname{LRRCP}(-1)$ & & \\
\hline LRSUB(-1) & & & LRSUB(-1) & $\begin{array}{c}0.459 \\
(0.345)\end{array}$ & \\
\hline LTRA & $\begin{array}{c}0.153 \\
(0.138)\end{array}$ & & DUM97 & $\begin{array}{c}-0.419 * * \\
(0.154)\end{array}$ & $\begin{array}{c}-0.622 * * * \\
(0.157)\end{array}$ \\
\hline $\operatorname{LTRA}(-1)$ & & & $\operatorname{ECM}(-1)$ & & $\begin{array}{c}-0.313 * * * \\
(0.060)\end{array}$ \\
\hline DUM74 & $\begin{array}{c}-0.037 \\
(0.161)\end{array}$ & & & & \\
\hline DUM79 & $\begin{array}{c}-0.084 \\
(0.152)\end{array}$ & & & & \\
\hline DUM87 & $\begin{array}{c}0.154 \\
(0.116)\end{array}$ & & & & \\
\hline DUM97 & $\begin{array}{c}-0.074 \\
(0.161)\end{array}$ & & & & \\
\hline DUM98 & $\begin{array}{c}-0.151 \\
(0.172)\end{array}$ & & & & \\
\hline $\bar{R}^{2}$ & 0.971 & 0.971 & $\bar{R}^{2}$ & 0.458 & 0.545 \\
\hline SE & 0.139 & 0.140 & SE & 0.125 & 0.115 \\
\hline $\mathrm{SC}(2)$ & $6.932 * *$ & 3.079 & $\mathrm{SC}(2)$ & 2.037 & 0.738 \\
\hline $\mathrm{FF}(1)$ & $6.012 * *$ & 0.793 & $\mathrm{FF}(1)$ & 0.294 & 0.009 \\
\hline $\mathrm{NO}(2)$ & 0.374 & 1.448 & $\mathrm{NO}(2)$ & 2.163 & 0.015 \\
\hline $\mathrm{HE}(1)$ & 15.358 & 5.032 & $\mathrm{HE}(1)$ & 4.098 & 3.404 \\
\hline
\end{tabular}

Note: see Tables 1 and 3.

ADLM are then used to test for cointegration relationships and estimate the corresponding ECMs.

To evaluate model performance, various diagnostic tests are carried out on each specific model. In addition, the ex post forecasting performance is checked for the specific models for Australia, Japan and the USA, in terms of mean absolute percentage error (MAPE) and root mean square error (RMSE). The results are shown in Tables 3-9.

The well-performing models are employed to forecast tourist arrivals up to 
Table 7. Estimates of Singapore models (1968-2000).

Dependent variable: LTOU

Partial Reduced ADLM adjustment
Dependent variable: DLTOU

WB-ECM JML-ECM

\begin{tabular}{|c|c|c|c|c|c|}
\hline INTECEPT & $\begin{array}{c}-0.785 \\
(0.797)\end{array}$ & $\begin{array}{c}-0.509 \\
(0.726)\end{array}$ & INTECEPT & $\begin{array}{c}-0.089 \\
(1.228)\end{array}$ & $\begin{array}{r}-0.077 \\
(0.058)\end{array}$ \\
\hline $\operatorname{LTOU}(-1)$ & $\begin{array}{l}0.779 * * * \\
(0.070)\end{array}$ & $\begin{array}{l}0.847 * * * \\
(0.049)\end{array}$ & DLGDP & $\begin{array}{c}0.071 \\
(0.885)\end{array}$ & $\begin{array}{c}-1.001 \\
(0.850)\end{array}$ \\
\hline LGDP & $\begin{array}{c}0.275 \\
(0.222)\end{array}$ & & DLRRCP & $\begin{array}{c}-1.025 * * \\
(0.470)\end{array}$ & $\begin{array}{c}-0.588 \\
(0.350)\end{array}$ \\
\hline LGDP(-1) & & & DLRSUB & $\begin{array}{c}0.728 \\
(0.426)\end{array}$ & \\
\hline LRRCP & $\begin{array}{c}-0.738^{* *} \\
(0.349)\end{array}$ & $\begin{array}{c}-0.879 * * * \\
(0.305)\end{array}$ & DLTRA & $\begin{array}{c}0.190 \\
(0.130)\end{array}$ & $\begin{array}{l}0.317 * * \\
(0.126)\end{array}$ \\
\hline $\operatorname{LRRCP}(-1)$ & & & LTOU $(-1)$ & $\begin{array}{c}-0.173 * \\
(0.096)\end{array}$ & \\
\hline LRSUB & $\begin{array}{l}0.800^{* * *} \\
(0.225)\end{array}$ & $\begin{array}{l}0.612 \text { *** } \\
(0.200)\end{array}$ & $\operatorname{LGDP}(-1)$ & $\begin{array}{c}0.145 \\
(0.297)\end{array}$ & \\
\hline $\operatorname{LRSUB}(-1)$ & & & $\operatorname{LRRCP}(-1)$ & $\begin{array}{c}-0.514 \\
(0.511)\end{array}$ & \\
\hline LTRA & $\begin{array}{c}0.127 \\
(0.085)\end{array}$ & $\begin{array}{l}0.169 * * * \\
(0.060)\end{array}$ & LRSUB(-1) & $\begin{array}{c}0.484^{*} \\
(0.280)\end{array}$ & \\
\hline $\operatorname{LTRA}(-1)$ & & & LTRASI(-1) & $\begin{array}{c}0.101 \\
(0.111)\end{array}$ & \\
\hline DUM74 & $\begin{array}{c}0.074 \\
(0.102)\end{array}$ & & DUM97 & $\begin{array}{c}0.303^{*} \\
(0.149)\end{array}$ & $\begin{array}{c}0.190 \\
(0.120)\end{array}$ \\
\hline DUM79 & $\begin{array}{c}0.080 \\
(0.095)\end{array}$ & & $\operatorname{ECM}(-1)$ & & $\begin{array}{c}-0.214 * * * \\
(0.065)\end{array}$ \\
\hline DUM87 & $\begin{array}{c}0.065 \\
(0.072)\end{array}$ & & & & \\
\hline DUM97 & $\begin{array}{c}0.133 \\
(0.108)\end{array}$ & & & & \\
\hline DUM98 & $\begin{array}{l}0.348 * * \\
(0.136)\end{array}$ & $\begin{array}{l}0.299 * * \\
(0.121)\end{array}$ & & & \\
\hline $\bar{R}^{2}$ & 0.994 & 0.994 & $\bar{R}^{2}$ & 0.435 & 0.356 \\
\hline SE & 0.088 & 0.088 & SE & 0.091 & 0.098 \\
\hline $\mathrm{SC}(2)$ & 0.958 & 1.490 & $\mathrm{SC}(2)$ & 3.831 & 4.615 \\
\hline $\mathrm{FF}(1)$ & $12.529 * * *$ & 1.712 & $\mathrm{FF}(1)$ & 0.354 & 3.523 \\
\hline $\mathrm{NO}(2)$ & 0.236 & 0.207 & $\mathrm{NO}(2)$ & 0.556 & 0.920 \\
\hline $\mathrm{HE}(1)$ & 18.491 & 4.700 & $\operatorname{HE}(1)$ & 17.087 & 4.839 \\
\hline
\end{tabular}

Note: see Tables 1 and 3 .

2010, with an ARIMA model acting as the benchmark. The orders of the ARIMA models are decided by the Bayesian Information Criterion (BIC). The results are as follows: ARIMA $(1,1,0)$ for Australia, Japan, Korea and the USA, $\operatorname{ARIMA}(1,1,1)$ for the $U K, \operatorname{ARIMA}(2,1,0)$ for Malaysia, and $\operatorname{ARIMA}(2,1,2)$ for Singapore. The forecasts for the accepted specific models for each of the origin countries, together with the reduced ADLM, error correction and benchmark ARIMA models, are generated and the average growth rates of tourist arrivals over the period up to 2010 are calculated and presented in Table 10. These 
Table 8. Estimates of UK models (1963-2000).

\begin{tabular}{|c|c|c|c|c|c|c|c|}
\hline & \multicolumn{3}{|c|}{ Dependent variable: LTOU } & & \multicolumn{3}{|c|}{ Dependent variable: DLTOU } \\
\hline & $\begin{array}{c}\text { Partial } \\
\text { adjustment }\end{array}$ & $\begin{array}{c}\text { Dead } \\
\text { start }\end{array}$ & $\begin{array}{l}\text { Reduced } \\
\text { ADLM }\end{array}$ & & WB-ECM & $\begin{array}{l}\text { JML- } \\
\text { ECM1 }\end{array}$ & $\begin{array}{l}\text { JML- } \\
\text { ECM2 }\end{array}$ \\
\hline INTECEPT & $\begin{array}{l}-7.791 * * * \\
(2.196)\end{array}$ & $\begin{array}{c}-12.272 * * * \\
(2.705)\end{array}$ & $\begin{array}{c}-12.064 * * * \\
(2.232)\end{array}$ & INTECEPT & $\begin{array}{c}-12.258^{* * *} \\
(2.272)\end{array}$ & $\begin{array}{c}-0.079 * \\
(0.043)\end{array}$ & $\begin{array}{l}0.106 * * * \\
(0.026)\end{array}$ \\
\hline $\operatorname{LTOU}(-1)$ & $\begin{array}{l}0.667 * * * \\
(0.126)\end{array}$ & $\begin{array}{l}0.520 * * * \\
(0.126)\end{array}$ & $\begin{array}{l}0.410 * * * \\
(0.109)\end{array}$ & DLGDP & & $\begin{array}{c}-0.140 \\
(0.858)\end{array}$ & $\begin{array}{c}1.218 \\
(0.796)\end{array}$ \\
\hline LGDP & $\begin{array}{l}1.739 * * * \\
(0.549)\end{array}$ & & & DLRRCP & $\begin{array}{c}-0.222 \\
(0.143)\end{array}$ & $\begin{array}{c}-0.827^{*} \\
(0.418)\end{array}$ & $\begin{array}{c}-0.474 \\
(0.369)\end{array}$ \\
\hline $\operatorname{LGDP}(-1)$ & & $\begin{array}{l}2.583 * * * \\
(0.611)\end{array}$ & $\begin{array}{l}2.904 * * * \\
(0.514)\end{array}$ & DLRSUB & & $\begin{array}{c}0.633 \\
(0.406)\end{array}$ & $\begin{array}{c}0.301 \\
(0.366)\end{array}$ \\
\hline LRRCP & $\begin{array}{l}-0.296 \\
(0.426)\end{array}$ & & $\begin{array}{c}-0.244^{*} \\
(0.138)\end{array}$ & $\operatorname{LTOU}(-1)$ & $\begin{array}{l}-0.570 * * * \\
(0.114)\end{array}$ & & \\
\hline $\operatorname{LRRCP}(-1)$ & & $\begin{array}{c}-0.678 \\
(0.426)\end{array}$ & & $\operatorname{LGDP}(-1)$ & $\begin{array}{l}2.820 * * * \\
(0.535)\end{array}$ & & \\
\hline LRSUB & $\begin{array}{c}0.381 \\
(0.442)\end{array}$ & & & $\operatorname{LRRCP}(-1)$ & $\begin{array}{c}-0.456 \\
(0.352)\end{array}$ & & \\
\hline LRSUB $(-1)$ & & $\begin{array}{c}0.825 * \\
(0.443)\end{array}$ & $\begin{array}{l}0.330 * * \\
(0.145)\end{array}$ & $\operatorname{LRSUB}(-1)$ & $\begin{array}{c}0.538 \\
(0.348)\end{array}$ & & \\
\hline LTRA & & & & DUM97 & $\begin{array}{c}-0.163 \\
(0.103)\end{array}$ & $\begin{array}{c}-0.119 \\
(0.102)\end{array}$ & $\begin{array}{c}-0.253 * * \\
(0.099)\end{array}$ \\
\hline $\operatorname{LTRA}(-1)$ & & & & $\operatorname{ECM}(-1)$ & & $\begin{array}{l}-0.425 * * * \\
(0.083)\end{array}$ & $\begin{array}{c}-0.528 * * * \\
(0.090)\end{array}$ \\
\hline DUM74 & $\begin{array}{c}0.022 \\
(0.121)\end{array}$ & $\begin{array}{c}-0.169 \\
(0.126)\end{array}$ & & & & & \\
\hline DUM79 & $\begin{array}{c}0.083 \\
(0.117)\end{array}$ & $\begin{array}{c}0.048 \\
(0.104)\end{array}$ & & & & & \\
\hline DUM87 & & & & & & & \\
\hline DUM97 & $\begin{array}{c}-0.169 \\
(0.114)\end{array}$ & $\begin{array}{c}-0.129 \\
(0.109)\end{array}$ & $\begin{array}{c}-0.184^{*} \\
(0.097)\end{array}$ & & & & \\
\hline DUM98 & $\begin{array}{c}0.076 \\
(0.115)\end{array}$ & $\begin{array}{c}-0.0001 \\
(0.109)\end{array}$ & & & & & \\
\hline $\bar{R}^{2}$ & 0.990 & 0.992 & 0.992 & $\bar{R}^{2}$ & 0.432 & 0.391 & 0.465 \\
\hline SE & 0.106 & 0.096 & 0.092 & SE & 0.092 & 0.096 & 0.090 \\
\hline $\mathrm{SC}(2)$ & 4.342 & 5.039 & 2.230 & $\operatorname{sC}(2)$ & 4.498 & 3.508 & 3.508 \\
\hline $\mathrm{FF}(1)$ & 1.357 & 2.699 & 1.594 & $\mathrm{FF}(1)$ & 0.037 & 0.322 & 0.915 \\
\hline $\mathrm{NO}(2)$ & 3.541 & 4.045 & 0.604 & $\mathrm{NO}(2)$ & 0.838 & 1.993 & 1.467 \\
\hline $\mathrm{HE}(1)$ & 4.177 & 5.224 & 12.042 & $\mathrm{HE}(1)$ & 8.512 & 6.382 & 9.305 \\
\hline
\end{tabular}

Note: see Tables 1 and 3 .

forecast growth rates are compared with the historical growth rates over the last five and ten years. Also, the forecasts for the best performing models as judged by various diagnostic statistics, ex post forecasting performance, growth rate consistency among the models, and consistency with historic growth experience, are plotted in Figures 1-7, with the benchmark ARIMA forecasts.

\section{Australia}

Australia's data fit a number of the specific models. The autoregressive, growth rate, partial adjustment and dead start models all pass the restriction tests, and 
Table 9. Estimates of USA models (1968-95).

\begin{tabular}{|c|c|c|c|c|c|c|}
\hline & \multicolumn{3}{|c|}{ Dependent variable: LTOU } & \multicolumn{3}{|c|}{ Dependent variable: DLTOU } \\
\hline & $\begin{array}{c}\text { Dead } \\
\text { start }\end{array}$ & $\begin{array}{c}\text { Partial } \\
\text { adjustment }\end{array}$ & $\begin{array}{l}\text { Reduced } \\
\text { ADLM }\end{array}$ & & WB-ECM & JML-ECM \\
\hline INTECEPT & $\begin{array}{l}3.262 * * * \\
(0.961)\end{array}$ & $\begin{array}{l}2.576 * * \\
(1.044)\end{array}$ & $\begin{array}{l}2.257 * * * \\
(0.758)\end{array}$ & INTECEPT & $\begin{array}{l}2.257 * * * \\
(0.758)\end{array}$ & $\begin{array}{c}-0.011 \\
(0.018)\end{array}$ \\
\hline LTOU $(-1)$ & $\begin{array}{l}0.421 * * * \\
(0.099)\end{array}$ & $\begin{array}{l}0.507 * * * \\
(0.113)\end{array}$ & $\begin{array}{l}0.436 * * * \\
(0.095)\end{array}$ & DLGDP & & \\
\hline LGDP & & & & DLRRCP & & $\begin{array}{c}-0.111 \\
(0.273)\end{array}$ \\
\hline $\operatorname{LGDP}(-1)$ & & & & DLRSUB & & \\
\hline LRRCP & & $\begin{array}{c}-0.798 * * \\
(0.367)\end{array}$ & & DLTRA & & $\begin{array}{c}-0.050 \\
(0.118)\end{array}$ \\
\hline $\operatorname{LRRCP}(-1)$ & $\begin{array}{c}-0.826 * * \\
(0.364)\end{array}$ & & $\begin{array}{l}-1.204 * * * \\
(0.216)\end{array}$ & $\operatorname{LTOU}(-1)$ & $\begin{array}{l}-0.564 * * * \\
(0.095)\end{array}$ & \\
\hline LRSUB & & $\begin{array}{c}-0.181 \\
(0.400)\end{array}$ & & LGDP(-1) & & \\
\hline LRSUB $(-1)$ & $\begin{array}{c}-0.443 \\
(0.398)\end{array}$ & & & $\operatorname{LRRCP}(-1)$ & $\begin{array}{c}-1.204 * * * \\
(0.216)\end{array}$ & \\
\hline LTRA & & $\begin{array}{l}0.160 * * \\
(0.056)\end{array}$ & & LRSUB $(-1)$ & & \\
\hline $\operatorname{LTRA}(-1)$ & $\begin{array}{l}0.169 * * * \\
(0.047)\end{array}$ & & $\begin{array}{l}0.177 * * * \\
(0.040)\end{array}$ & $\operatorname{LTRA}(-1)$ & $\begin{array}{l}0.177 * * * \\
(0.040)\end{array}$ & \\
\hline DUM74 & $\begin{array}{c}0.126 \\
(0.091)\end{array}$ & $\begin{array}{c}0.209 * \\
(0.103)\end{array}$ & & DUM79 & $\begin{array}{l}-0.167 * * \\
(00072)\end{array}$ & $\begin{array}{r}-0.147 * \\
(0.073)\end{array}$ \\
\hline DUM79 & $\begin{array}{c}-0.159 * * \\
(0.072)\end{array}$ & $\begin{array}{c}-0.206 * * \\
(0.084)\end{array}$ & $\begin{array}{c}-0.167 * * \\
(0.072)\end{array}$ & $\operatorname{ECM}(-1)$ & & $\begin{array}{r}-0.532 * * * \\
(0.085)\end{array}$ \\
\hline DUM87 & $\begin{array}{c}0.082 \\
(0.055)\end{array}$ & $\begin{array}{c}0.068 \\
(0.062)\end{array}$ & & & & \\
\hline $\bar{R}^{2}$ & 0.968 & 0.956 & 0.967 & $\bar{R}^{2}$ & 0.625 & 0.620 \\
\hline SE & 0.068 & 0.079 & 0.069 & SE & 0.069 & 0.069 \\
\hline $\mathrm{SC}(2)$ & 3.031 & 5.197 & 1.420 & $\mathrm{SC}(2)$ & 1.420 & 1.814 \\
\hline $\mathrm{FF}(1)$ & 1.164 & $5.723 * *$ & 0.425 & $\mathrm{FF}(1)$ & 3.749 & \\
\hline $\mathrm{NO}(2)$ & 0.262 & 0.227 & 0.222 & $\mathrm{NO}(2)$ & 0.222 & 0.351 \\
\hline $\mathrm{HE}(1)$ & 14.402 & $20.888 * *$ & 8.677 & $\mathrm{HE}(1)$ & 8.677 & 3.279 \\
\hline $\operatorname{PF}(d f)$ & 0.971 & 0.608 & 0.959 & $\operatorname{PF}(d f)$ & 0.959 & 0.657 \\
\hline MAPE & 0.698 & 0.335 & 0.727 & MAPE & 0.727 & 0.930 \\
\hline RMSE & 0.106 & 0.063 & 0.110 & RMSE & 0.110 & 0.126 \\
\hline
\end{tabular}

Note: see Tables 1 and 3 .

each of these restricted models passes all the diagnostic checks. With regard to ex post forecasting accuracy, the dead start and partial adjustment models perform best, and are therefore selected as the specific models for the ex ante forecasts (see Table 3). The reduced ADLM shows that the lagged dependent variable, current income, lagged own price and lagged substitute prices are all key factors in motivating Australian tourists to travel to Thailand. One CI relationship is detected by the Johansen CI test, and the corresponding JMLECM is estimated together with the WB-ECM.

Concerning the ex ante forecast performance of each model, Table 10 shows that, apart from the ARIMA model, all the other five models forecast an obvious 
Table 10. Forecast average growth rates of tourist arrivals (2001-10) from various models compared with historical records $(\%)$.

\begin{tabular}{lccccccc}
\hline & Australia & Japan & Korea & Malaysia & Singapore & UK & USA \\
& & & & & & & \\
ARIMA & 0.45 & 1.59 & 2.62 & 2.06 & 9.15 & 9.47 & 0.98 \\
Partial adjustment & 12.55 & - & 21.03 & 9.60 & 7.93 & 11.39 & 3.72 \\
Dead start/Growth rate* & 11.86 & 7.33 & 23.06 & - & - & 11.81 & 4.31 \\
Reduced ADLM & 14.07 & 7.89 & 21.75 & 2.61 & 8.80 & 11.42 & 4.83 \\
WB-ECM & 14.45 & 7.42 & 22.27 & 10.14 & 7.45 & 11.57 & 4.83 \\
JML-ECM1 & 7.12 & 12.65 & 20.66 & 20.43 & -2.60 & 21.00 & 13.42 \\
JML-ECM2 & - & - & - & - & - & 24.58 & - \\
Actual rate (1990-2000) & 2.51 & 6.27 & 11.73 & 3.44 & 6.93 & 7.65 & 5.19 \\
Actual rate (1995-2000) & 10.92 & 8.02 & -0.37 & -0.42 & 8.77 & 11.67 & 10.64 \\
\hline
\end{tabular}

* The growth rate model concerns the Korean case.

upward trend of tourist arrivals, with a relatively lower growth rate for the JML-ECM. The historical data show that in the last ten years tourist arrivals from Australia to Thailand experienced only $2.5 \%$ annual growth on average, but during the last five years the growth has been accelerating up to $10.9 \%$ on average. On the basis of ex post forecasting performance, the dead start model is likely to generate the most accurate forecasts, followed by the partial adjustment model. Also these models generate close forecasts, which are consistent with

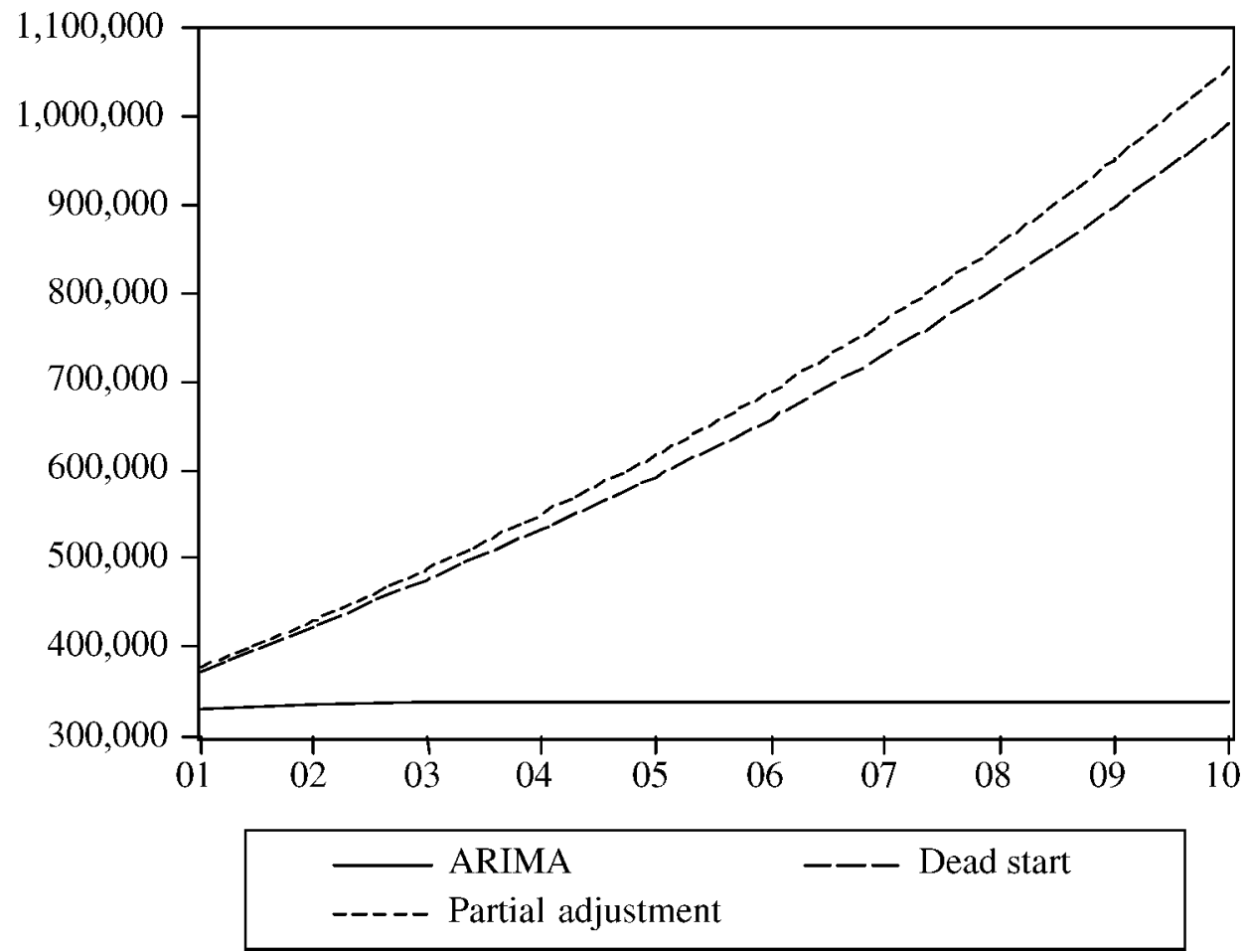

Figure 1. Forecasts of tourist arrivals from Australia. 
growth in the recent past. The results from the dead start model are therefore taken to be the most realistic, with 992,000 tourist arrivals in 2010 projected, growing $11.9 \%$ each year on average.

\section{Japan}

Only the dead start model passes the restriction tests in the Japanese case (see Table 4). Both the dead start model and the reduced ADLM confirm that the two oil crises have had adverse effects on Japanese outbound tourism demand, which is also validated by the two ECMs. One CI relationship is found among tourist arrivals, own price and substitute prices. All the models pass the various diagnostic tests, except for the JML-ECM, which fails only the heteroscedasticity test at the $5 \%$ significance level, but passes it at the $1 \%$ level. Concerning the ex post forecasts, the dead start model, reduced ADLM and the WB-ECM perform similarly, all being superior to the JML-ECM.

Table 10 shows that the dead start model, the WB-ECM and the reduced ADLM produce similar ex ante forecasts, all with average annual growth rates between $7 \%$ and $8 \%$. The ARIMA model and the JML-ECM generate extremely conservative and over-optimistic forecasts, respectively, compared with historical growth records, which shows increases of 6.3\% during 1990-2000 and 8.0\% during 1995-2000. The reduced ADLM is the most reliable model according to the ex post accuracy measures, and it shows that tourist arrivals are expected to rise to 2.56 million in 2010 .

\section{Korea}

The growth rate and partial adjustment models fit the Korean data best as only these two models pass the restriction tests. As seen in Table 5, the second oil crisis and the Asian financial crisis display significant adverse impacts, as expected. Both DUM97 and DUM98 are significant, indicating that the negative influence of the Asian financial crisis on Korean tourism demand in Thailand has been persistent during the whole process. The influence took effect through the collapse of Thai travel agents in Korea, as mentioned earlier. In addition to the lagged dependent variable, income and substitute prices also show their significant importance during the decision making process. The WB-ECM and one JML-ECM are estimated since one CI relationship exists among the demand variables.

In terms of the ex ante forecasts (Table 10), all the econometric models generate similar forecast growth rates, whereas the ARIMA model predicts a much lower growth rate. According to the medium forecasts from the reduced ADLM, tourist arrivals from Korea to Thailand are expected to increase to 3.20 million in 2010, growing $21.8 \%$ each year. This forecast growth rate is much higher than during the last few years.

\section{Malaysia}

The partial adjustment model is accepted by the Malaysian data, and passes all the diagnostic tests at the $1 \%$ level (see Table 6). The estimates of the reduced ADLM show that substitute prices are the major consideration of Malaysian tourists when faced with the tourism decision. The estimates of the two ECMs 


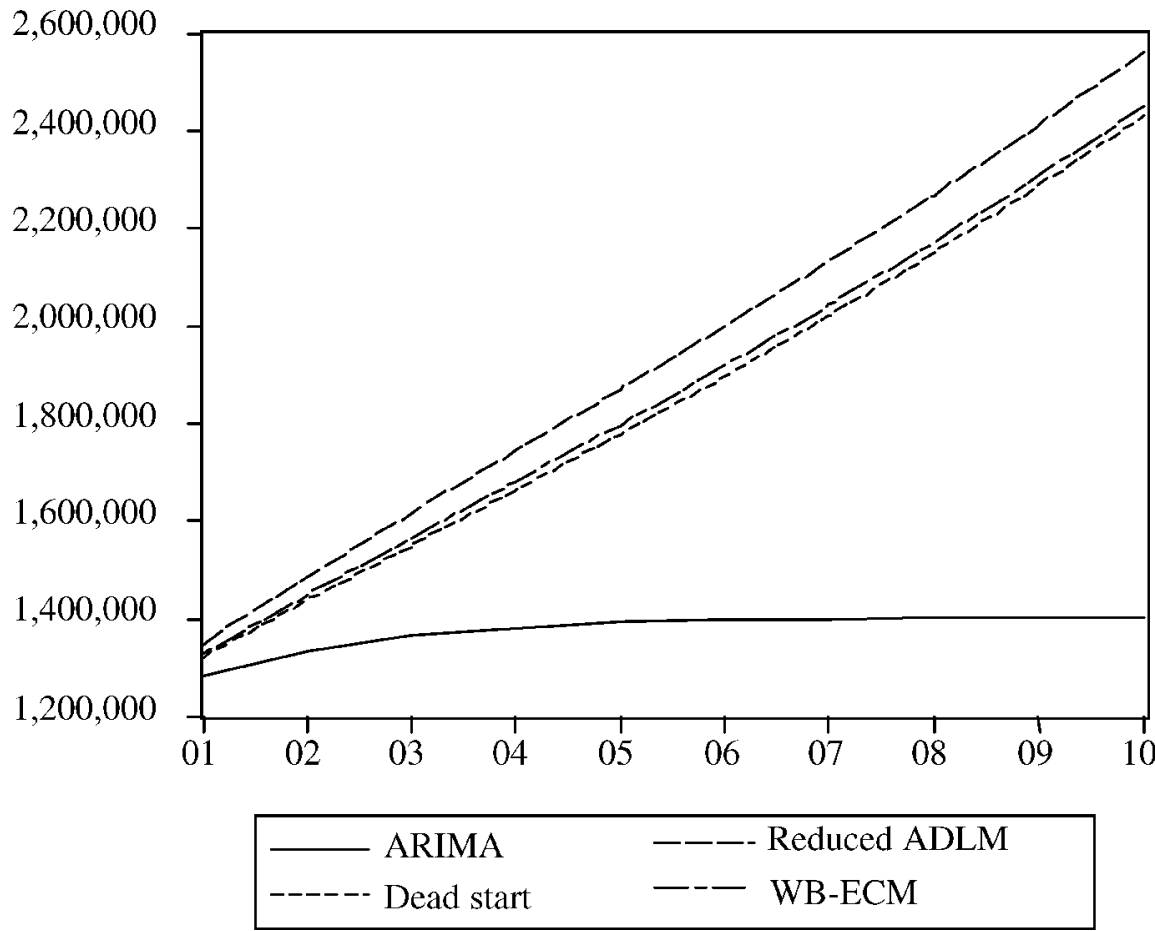

Figure 2. Forecasts of tourist arrivals from Japan.

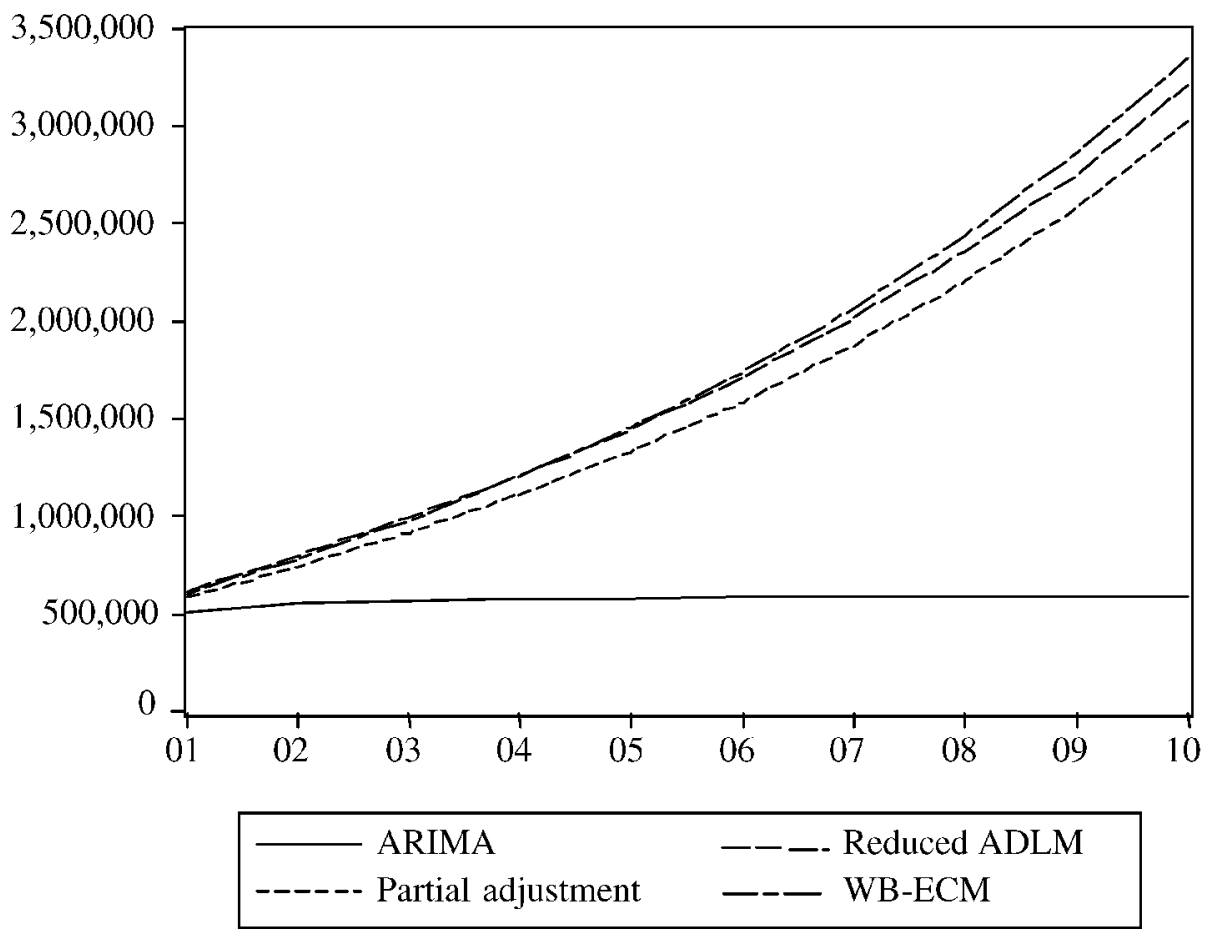

Figure 3. Forecasts of tourist arrivals from Korea. 


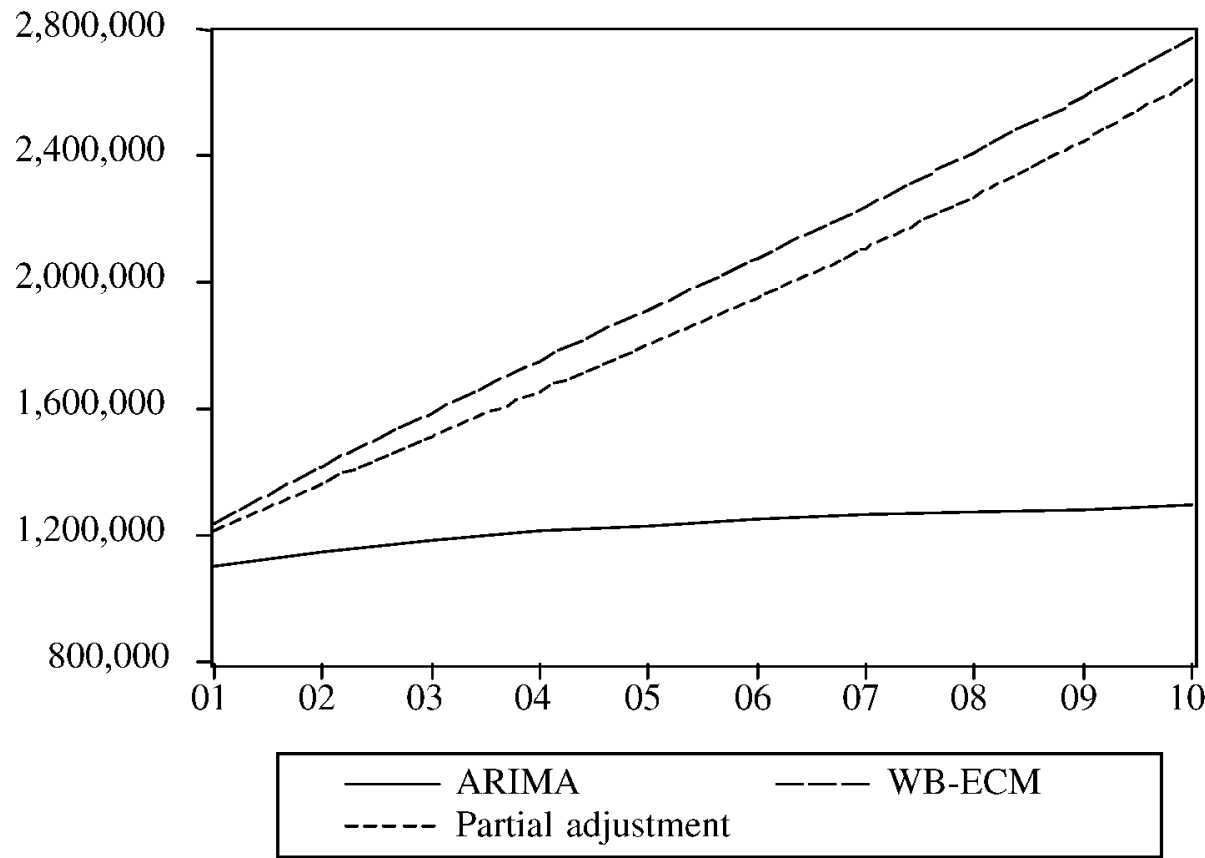

Figure 4. Forecasts of tourist arrivals from Malaysia.

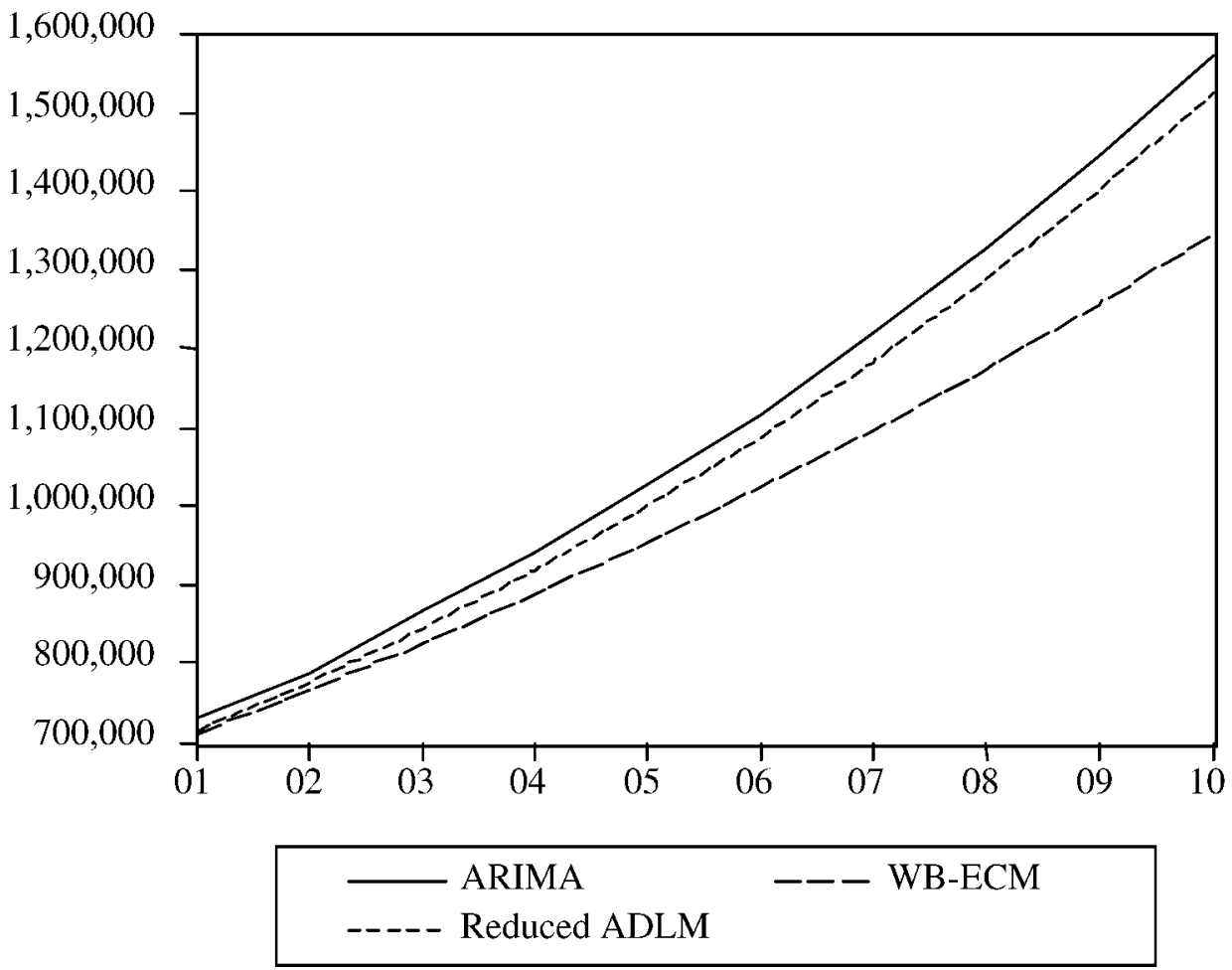

Figure 5. Forecasts of tourist arrivals from Singapore. 

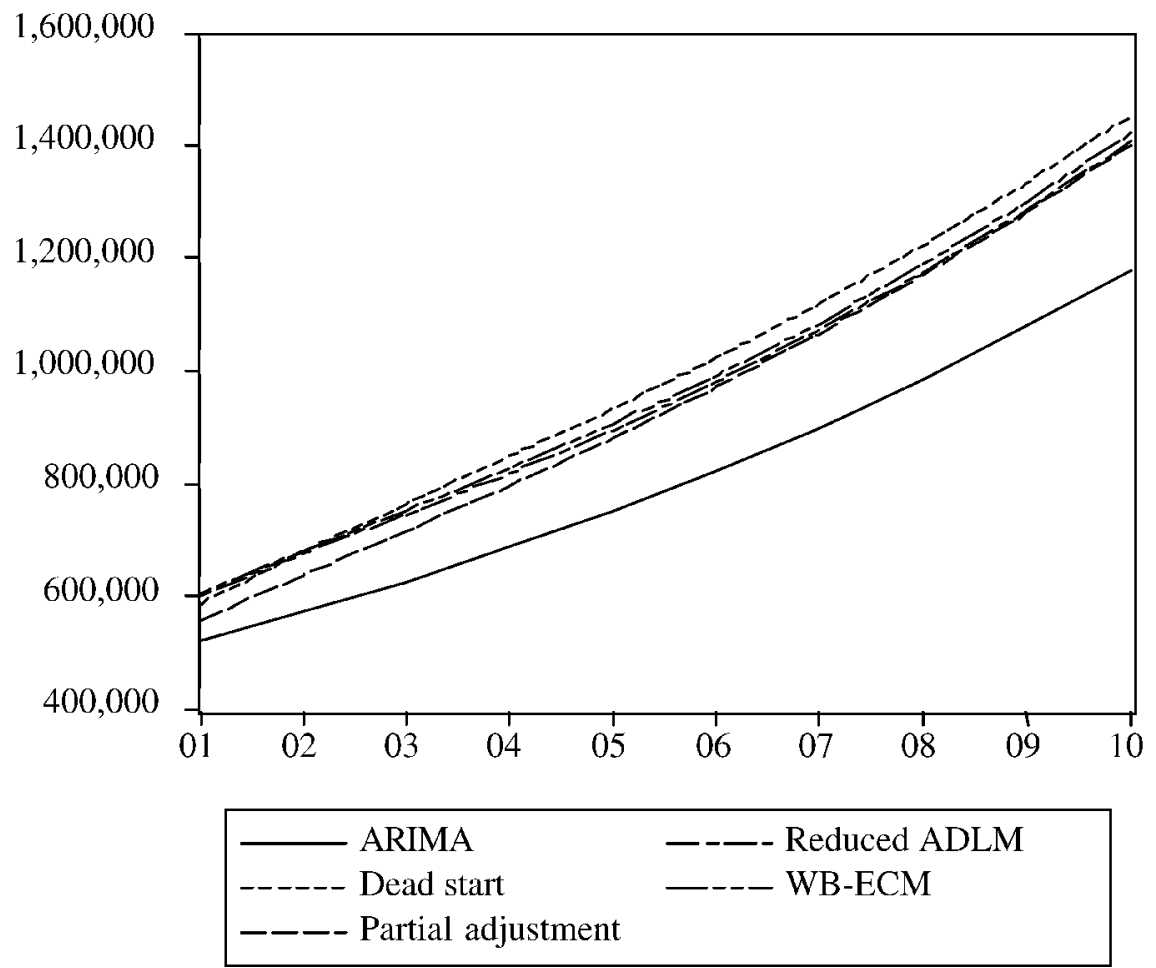

Figure 6. Forecasts of tourist arrivals from the UK.

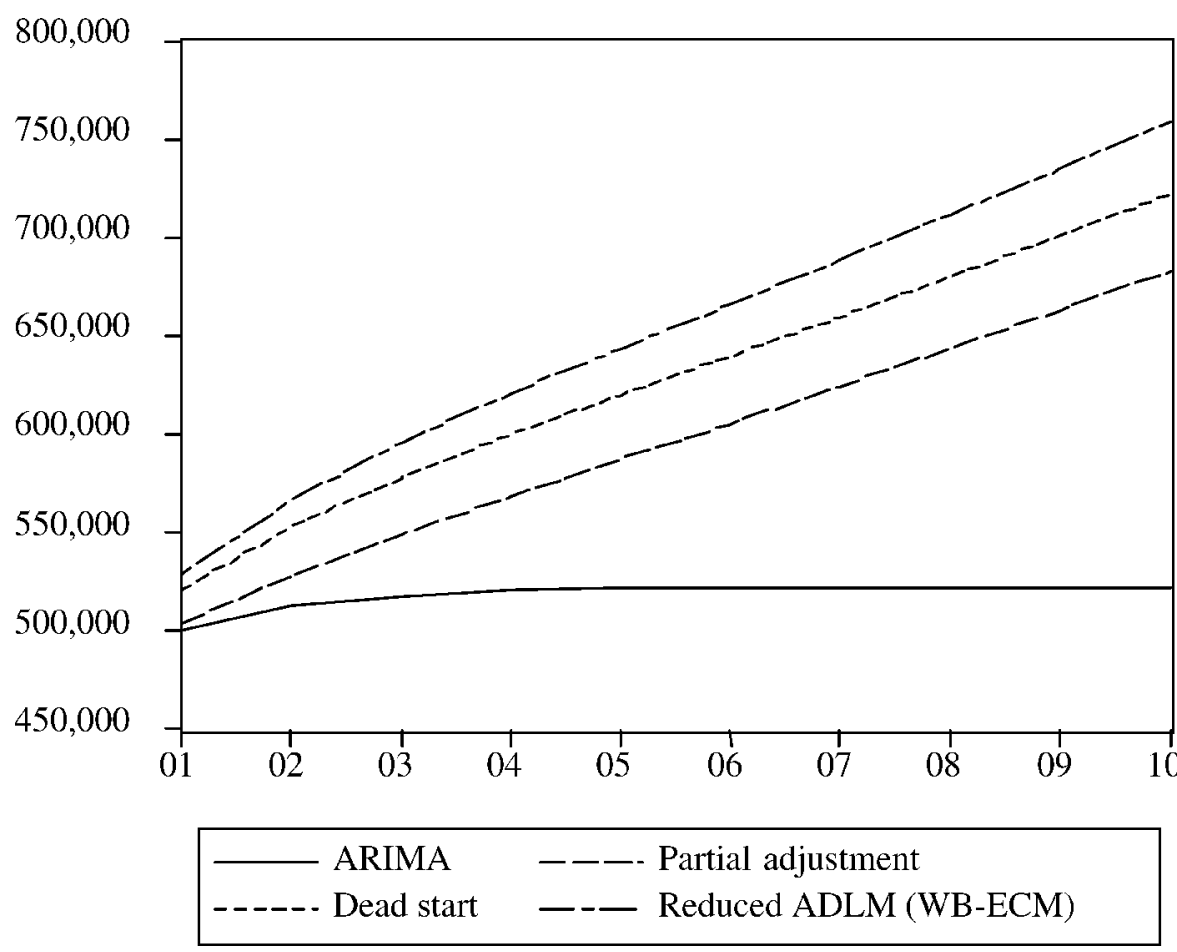

Figure 7. Forecasts of tourist arrivals from the USA. 
show that the financial crisis had a significant impact on the demand for Thai tourism by Malaysian residents, although this impact is apparent only in 1997 (not 1998). The adverse effect is associated with the slowing-down growth of income in the early stages of the crisis.

With regard to the ex ante forecasts (Table 10), three levels of predictions are achieved $(2-3 \%, 10 \%$ and $20 \%)$. Only one econometric model predicts in the $2-3 \%$ range and only one model at $20 \%$ per year, whereas two econometric models forecast $10 \%$ average growth per year. Also, considering the growth during the last decade ( $3.4 \%$ on average), along with the recovery from the financial crisis, an annual increase of about $10 \%$ in the medium future seems reasonable. Given that the diagnostic test results are better for the WB-ECM than the partial adjustment model, tourist arrivals from Malaysia are expected to rise to 2.77 million in 2010 , as suggested by the WB-ECM.

\section{Singapore}

The reduced ADLM presents a functional form that is consistent with the partial adjustment model, which is the only specific model that passes the restriction tests (see Table 7). Due to its better performance judged by the diagnostic tests, the reduced ADLM is employed for the ex ante forecasts. In the reduced ADLM, apart from the income variable, all other variables are found to be statistically significant, including trade volume which does not display significance for any other origins. Regarding the Asian financial crisis, Singapore is the only country to benefit from it in the tourism context, and this is confirmed by three out of four specific models. One possible reason for this positive impact on the demand for Thai tourism by Singaporeans is due to the much less severe devaluation of the Singapore dollar. One CI relationship is detected and two ECMs are estimated, using the WB and JML methods. Both of the ECMs pass all the diagnostic tests.

With regard to the ex ante forecasts (Table 10), the JML-ECM comes out with an unreasonable downturn trend. This is in contrast to all the other models, where the results are quite similar. According to the reduced ADLM, it is likely that tourist arrivals from Singapore will increase to 1.52 million in 2010.

\section{$U K$}

The partial adjustment and dead start models are appropriate functional forms for the UK data (see Table 8). The reduced ADLM indicates that income and substitute prices are the key determinants for UK tourism demand to Thailand. DUM97 also appears in the final reduced model with a negative sign, implying that the Asian financial crisis had an adverse impact on UK tourism to this destination (maybe even the whole region) in the early stages. Examination of tourist arrivals from the UK between 1996 and 1997 shows that, although tourist numbers kept increasing from 286,889 to 287,664, the growth rate slowed from $4.6 \%$ to $2.7 \%$. It seems that although the devaluation caused a more attractive tourism price, other negative factors played more important roles. For example, tourists might have worried more about political stability and personal security. With the development of the financial crisis the positive and negative factors tended to offset each other, so the overall influence was neutral in 1998. The CI test indicates two CI relationships, which are both 
correctly signed, and therefore the corresponding two JML-ECMs are employed to generate ex ante forecasts, along with the WB-ECM.

Regarding the ex ante forecasts (Table 10), all the models come out with forecasts that show an upward trend. Apart from the two JML-ECMs, which forecast average annual growth rates of $21-25 \%$, the other four econometric models generate very close forecasts, with average annual growth rates in the range $11-12 \%$, and this is close to the historical rate over the last five years. The reasonable predicted ranges for tourist arrivals based on these models are from 1.40 million to 1.46 million in 2010 .

\section{USA}

The partial adjustment and dead start models are acceptable functional forms for the USA, the same as in the cases of Australia and UK (see Table 9). In the reduced ADLM, besides the lagged dependent variable, lagged own price and lagged trade volume are also significant at the $1 \%$ level. The reduced ADLM coincides with the dead start specification. As for the one-off events, only the second oil crisis is statistically significant, and this is supported by all the other specific models. Like most of the other origins, only one CI relationship is traced. Since the impact of the Asian financial crisis is not detected in the models, the data for estimation are taken from before 1995 , and the ex post forecast performance is also checked. The results show that the partial adjustment model clearly exhibits the best ex post forecast performance.

When the ex ante forecasts are investigated (Table 10), the JML-ECM describes the most optimistic prospect, with $13.4 \%$ growth each year on average. By contrast, the ARIMA model predicts most conservatively, with only $1 \%$ growth being achieved. The partial adjustment model, dead start model, reduced ADLM and WB-ECM all generate forecast growth rates of around 4\%, which is close to the average annual growth rate over the period 1990-2000. The partial adjustment model generates the most accurate ex post forecasts, but has some problems with diagnostic tests. The dead start model generates the next most accurate ex post forecasts and is therefore used to forecast tourist arrivals. The dead start model suggests that 722,000 tourist arrivals are expected in 2010 .

Compared with the ARIMA models, most of the econometric models predict much higher forecasts, especially in the Australian and Korean cases. The reason for this is that the forecasts of the econometric models are influenced by both the historical trend of the demand variable itself, but also by the predictions of the future trends of the influencing factors. The forecasts of the ARIMA models, however, are influenced only by the historical trend of the forecast variable itself.

\section{Summary and conclusion}

The demand for Thai tourism by seven major origin countries is analysed using the general-to-specific modelling methodology. Various specific functional forms are examined in addition to the reduced ADLM, the Wickens-Breusch ECM and the Johansen maximum likelihood ECM. The forecasting models are selected based on a number of strict criteria including the goodness of fit, diagnostic 
statistics and ex post forecasting ability. The empirical results in this study suggest that the partial adjustment and dead start models are the most acceptable specific functional forms.

With regard to the reduced ADLM, habit persistence features in all the models except for Japan. Income is shown to be the key determinant in the cases of Australia, Korea and the UK. The own price and cross price variables play important roles during the decision making process of residents from Australia, Japan, Singapore and the UK. It should be noted that the sign of coefficients for $R S U B_{t}$ in the Korean and USA models is different from that in the other models, implying that neighbouring countries have played different roles when different origin countries are concerned. The negative sign suggests that the neighbouring destinations are complementary to Thailand, while the positive sign indicates that the substitute effect is in force. This result has an important implication for Thailand's tourism pricing strategies.

Trade volume turns out to be significant only in the cases of Singapore and the USA, perhaps because of the relatively high proportion of business tourism from these two countries. With regard to one-off events, the first oil crisis had a significant adverse impact in the Japan model, while the influence of the second oil crisis is significant in the Japan, Korea and USA models. The direction of influence of the Asian financial crisis on tourism demand differs from origin to origin. Singapore seems to gain from the cheaper price due to the relatively huge currency devaluation in Thailand, while Korea and the UK suffer from the crisis. The impact took effect in Korea in 1997 and continued into 1998. As for Singapore, a significant impact could be seen only in 1998, while in the case of the UK the effect was more evident at the beginning of the crisis.

With regard to the ex ante forecasts, the ARIMA model always produces the most modest prediction (with the exception of Singapore), while the JML-ECM tends to generate the most optimistic forecast. The most reliable forecast has been selected for each origin based on diagnostic tests, ex post forecasting performance, consistency among forecast growth rates from the various models, and consistency with historical growth over the last five to ten years. It appears that tourist arrivals from Korea will grow most rapidly among all the origin countries, and that growth from the UK will be the slowest. The forecasts also show that Korea and Malaysia will join Japan to become the largest tourism markets for Thailand by the end of this decade.

Since rapid growth in tourist arrivals from such origin countries as Australia, Japan, Korea and the UK is predicted, it is important for Thailand to increase flight frequencies between major Thai cities and these countries. The improvement of airport facilities and services in Thailand is also considered to be crucial for attracting more tourists from these countries, particularly during the peak seasons. The Thai government should encourage greater private-sector involvement, and especially investment in the development of tourism projects. Much attention should be paid to catering for the needs of Korean tourists, as Korea is predicted to be the fastest-growing market for Thailand. In addition, establishing more travel agencies or organizing broader cooperation with local agencies in Korea would be beneficial for Thai tourism. Moreover, the complementary interplay between Thailand and its neighbouring countries in the case of Korean and American tourists suggests that the development of joint 
touring programmes would increase tourism demand in Thailand as well as in the neighbouring countries. However, the government should not ignore the possible negative impacts of speedy tourism expansion on the physical and social environments within Thailand.

It should be noted that, due to the terrorist attacks on the USA on 11 September 2001, and the war on Iraq and the SARS epidemic in 2003, the demand for Thai tourism is likely to be affected. However, according to past experience the impacts are likely to be short-lived, and a full recovery should be achieved within the next two years (Louvieris, 2002). Therefore, the general trends in the demand for Thai tourism predicted in this study are unlikely to be affected much over the forecasting period.

\section{Endnotes}

1. These figures do not include overseas Thais.

2. Data source: TAT Website (www.tat.or.th).

3. By 'equilibrium demand' we mean that there is no over- or under-demand for tourism if the influencing factors of tourism demand are given.

4. A detailed explanation of the ARIMA modelling methodology can be found, for example, in Pankratz (1983).

5. The mainland of China, Taiwan and Germany are also important tourism origins, but due to data unavailability or insufficiency they are excluded from this study.

6. Data source: www.tat.org.

7. Raw data of tourist arrivals before 1978 were recorded only by nationality rather than by both nationality and residence as was the case afterwards. Since the latter is the proper measurement, and considering the high correlation between the two series, simple regression of tourist arrivals by residence against arrivals by nationality is used to estimate the missing data. In doing so, the full data set of tourist arrivals by residence is obtained.

8. The definition of the substitute price index follows that in the deduced demand equation of Thomas (1993). It differs slightly from the substitute price index used in Song et al (2000), Song and Witt (2000) and Kulendran and Witt (2001), where it is measured by price in the destination relative to that in competing destinations.

9. Apart from those mentioned, some other origin-specific events may also have affected the demand for Thai tourism. However, due to the restriction of degrees of freedom, they were not included.

\section{References}

Breusch, T. (1978), 'Testing for autocorrelation in dynamic linear models', Journal of Australian Economic Papers, Vol 17, pp 334-355.

Chow, G. (1960), 'Test of equality between coefficients in two linear regressions', Econometrica, Vol 28, pp 591-605.

Engle, R. F., and Granger, C. W. J. (1987), 'Cointegration and error correction: representation, estimation and testing', Econometrica, Vol 55, pp 251-276.

Gilbert, C. L. (1986), 'Professor Hendry's econometric methodology', Oxford Bulletin of Economics and Statistics, Vol 48, No 3, pp 283-307.

Godfrey, L. G. (1978), 'Testing for higher order serial correlation in regression equations when the regressors contain lagged dependent variables', Econometrica, Vol 46, pp 1303-1310.

Hendry, D. F. (1995), Dynamic Econometrics, Oxford University Press, Oxford.

Higham, J. (2000), 'Thailand: prospects for a tourism-led economic recovery', in Hall, C. M., and Page, S. (eds), Tourism in Southeast Asia: Issues and Cases, Butterworth-Heinemann, Oxford.

Jarque, C. M., and Bera, A. K. (1980), 'Efficient tests for normality, homoscedasticity and serial independence of regression residuals', Economic Letters, Vol 6, pp 255-259.

Johansen, S. (1988), 'A statistical analysis of cointegration vectors', Journal of Economic Dynamics and Control, Vol 12, pp 231-254. 
Kulendran, N., and Witt, S. F. (2001), 'Cointegration versus least squares regression', Annals of Tourism Research, Vol 28, pp 291-311.

Li, L. and Zhang, W. (1997), 'Thailand: the dynamic growth of Thai tourism', in Go, F. M., and Jenkins, C. L. (eds), Tourism and Economic Development in Asia and Australia, Cassell, London and Washington, DC.

Louvieris, P. (2002), 'Forecasting demand for Greece tourism', Journal of Travel and Tourism Marketing, Vol 13, No 1/2, pp 21-41.

Pankratz, A. (1983), Forecasting with Univariate Box-Jenkins Models, Wiley, New York.

Ramsey, J. B. (1969), 'Test for specification errors in classical linear least squares regression analysis', Journal of the Royal Statistical Society, Series B, Vol 31, pp 350-371.

Song, H., and Witt, S. F. (2000), Tourism Demand Modelling and Forecasting: Modern Econometric Approaches, Pergamon, Oxford.

Song, H., and Witt, S. F. (2003), 'Tourism forecasting: the general-to-specific approach', Journal of Travel Research, Vol 42, No 3, pp 65-74.

Song, H., Romilly, P. and Liu, X. (2000), 'An empirical study of outbound tourism demand in the UK', Applied Economics, Vol 32, pp 611-624.

Thomas, R. L. (1993), Introductory Econometrics: Theory and Applications, 2nd edition, Longman, London.

Vogt, M. G. and Wittayakorn, C. (1998), 'Determinants of the demand for Thailand's exports of tourism', Applied Economics, Vol 30, pp 711-715.

White, H. (1980), 'A heteroscedasticity-consistent covariance matrix estimator and a direct test of heteroscedasticity', Econometrica, Vol 48, pp 817-838.

Wickens, M. R., and Breusch, T. S. (1988), 'Dynamic specification, the long-run and the estimation of transformed regression model', Economic Journal, Vol 98 (Conference), pp 189-205.

Witt, S. F. (1980), 'An abstract mode-abstract (destination) node model of foreign holiday demand', Applied Economics, Vol 12, No 2, pp 163-180.

Witt, S. F., Song, H., and Louvieris, P. (2003), 'Statistical testing in forecasting model selection', Journal of Travel Research, Vol 42, No 3. 